\title{
Synchronie, Diachronie und - Futurität. Die französischen Futura: Kategorien und Methoden ihrer Beschreibung*
}

\section{Kategorien und Methoden: Linien der Argumentation}

Die 1999 erschienene Dissertation von Monika Sokol fokussiert erstmals systematisch die Interaktionen von Aspekt und aktionalen Strukturen bei den französischen Futura und spürt möglichen Affinitäten und Restriktionen dieses Zusammenspiels nach. ${ }^{1}$ Durch die Verbindung synchroner und diachroner Analysen konzentrieren sich in ihrer Untersuchung nicht nur zentrale Fragestellungen der Tempus- und Aspektforschung, die Studie wirkt darüber hinaus als Katalysator für grundlegende Reflexionen zu Prinzipien und Methoden der (romanistischen) Sprachwissenschaft.

Im Zentrum von Sokols Monographie steht die Frage, ob Affinitäten oder gar Restriktionen zwischen futurisch fungierenden Formen und aktionalen Klassen bestehen und ob es im Subsystem der Futura Aspektoppositionen gibt [6]. Sokols Ziel ist es, sowohl synchrone Funktionsmuster von futur simple (FS), futur périphrastique (FP) und présent futural (PF) im Französischen der Gegenwart als auch die diachrone Entwicklung dieser Formen über das Zusammenspiel von Aspekt und aktionalen Klassen zu erklären. Aspektualität und Aktionalität werden dabei als Kategorien verstanden, aus deren Interaktion temporale und modale Werte resultieren und die damit der Temporalität und der Modalität hierarchisch vorgeordnet sind [4, 16]. Dazu entwirft sie ein übereinzelsprachliches Modell der Kategorieninteraktion, dessen Anwendbarkeit anhand der französischen Futura exemplifiziert werden soll [3s.]. Diese Verbindung von Theoriebildung und einzelsprachlicher Analyse soll zwei Fehler vermeiden, die Sokol in sprachwissenschaftlichen Untersuchungen zu erkennen glaubt: so würden zu stark theorieorientierte Ansätze das Sprachmaterial oft reduktiv ausklammern, während zu stark am einzelsprachlichen Material orientierte Studien oft den theoretischen «Überbau» vermissen ließen und nur aus «statistisch-deskriptiver Fleißarbeit» bestünden [2]. Eine Kernfrage meiner Besprechung ist daher, inwiefern die von Sokol verwendete Kombination von übereinzelsprachlichen Regeln, diachronen Ausgriffen

* Besprechungsaufsatz zu: Monika Sokol, Das Zusammenspiel der Verbalkategorien und die französischen Futura, Tübingen, Niemeyer, 1999, VIII + 218 p.

${ }^{1}$ Für wertvolle Anregungen zu diesem Aufsatz bin ich Franz Lebsanft, Claus Morgenstern und Wolf-Dieter Stempel zu herzlichem Dank verpflichtet. Mein Dank gilt ferner Emmanuel Faure, Marguerite Gagneur, Sarah Brun und Josette Boyer, die mir ihre muttersprachliche Kompetenz zur Verfügung stellten. 
und synchroner Datenerhebung es erlaubt, Hypothesen zu Aspekt und aktionalen Strukturen nicht nur aufzustellen, sondern auch zu überprüfen.

Im Folgenden wird zunächst das Modell der aktionalen Klassen besprochen, wobei die Frage nach dem kognitiven Status dieser Klassen und damit auch nach dem Verhältnis von Sprach- und Kognitionswissenschaft von zentraler Bedeutung sein wird. Danach werden anhand der Ausgriffe in die diachrone Entwicklung von FS und FP Methoden und methodische Klippen des von Sokol verwendeten Ansatzes herausgearbeitet. Leitmotive werden dabei das Verhältnis von dokumentierter und konjizierter Sprachgeschichtsschreibung sowie das Spannungsverhältnis von einzelsprachlicher Sprachgeschichte und übereinzelsprachlichen Tendenzen der Sprachentwicklung sein. Da Sokol ihre Darstellung der synchronen Funktionsmuster der Futura als «Gegenstück und Ergänzung» [2] zu meiner 1997 erschienenen Monographie zur Semantik und Pragmatik der französischen Futura im Französischen der Gegenwart (Schrott 1997a) konzipiert, ist eine weitere Kernfrage meiner Untersuchung, ob die Funktionsmuster von FS und FP im Französischen der Gegenwart, wie Sokol annimmt, an die Interaktion von Aspekt und aktionalen Klassen gekoppelt sind oder ob hier andere semantische Charakteristika der Formen die Fäden ziehen. ${ }^{2}$ Im Vergleich mit dem von mir (1997a) präzisierend entwickelten «Prinzip der Konditionierung» soll die Erklärungskraft der beiden Modelle für die Futuropposition verglichen werden. In diesem Sinne versteht sich die vorliegende Besprechung als Fortsetzung des von Monika Sokol begonnenen Dialogs.

\section{Einzelsprachliche Äußerungen und kognitive Muster: aktionale Klassen}

Eine wichtige Vorarbeit für die Untersuchung der Interaktionen zwischen aktionaler Semantik und grammatischen Aspekten ist das von Sokol entworfene «Modell der verbalkategorialen Inhalte» [Kapitel 3, 31-79], das ein System aktionaler Muster entwirft. Für dieses Kategorienmodell wird als Basis ein semantisches Rahmenmodell etabliert [Kapitel 2, 19-29], das vier Ebenen umfaßt, wobei die Ebene 1 die Referenten der außersprachlichen Welt und die Ebene 2 die ihnen entsprechenden kognitiven Konzepte beinhaltet; die Ebenen 3 und 4 erfassen dagegen die sprachlichen Zeichen, und zwar die Inhaltsseite (Ebene 3) und die Ausdrucksseite des sprachlichen Zeichens (Ebene 4) (cf. die Graphiken [23] und [29]). ${ }^{3}$ Das Modell trägt damit der in der (historischen) Semantik allgemein akzeptierten Differenzierung zwischen kognitiven

\footnotetext{
${ }^{2}$ Bei meinen Ausführungen werde ich mich auf $F S$ und FP konzentrieren und auf das $P F$ nur insoweit eingehen, als es für die Opposition dieser beiden Formen eine Rolle spielt.

${ }^{3}$ Das Modell ist aus einem mittelalterlich-scholastischen Modell (nach Raible 1987) und einem generativen Zwei-Ebenen-Modell, dessen generativistische Grundannahmen jedoch ausgespart bleiben [19s.], kombiniert.
} 
Strukturen bzw. Konzepten und einzelsprachlichen Bedeutungen Rechnung, ${ }^{4}$ es hat aber als Zeichenmodell den Nachteil, daß es den Bereich der Rede im Kontext nur ungenau erfaßt. Denn der Bereich zwischen den sprachlichen Ebenen (3 und 4) und den außersprachlichen Ebenen (1 und 2) wird mit «Sinn/Bedeutung» [23] beschriftet, so daß diese Begriffe, die in der Romanistik in der Tradition Eugenio Coserius (cf. etwa Coseriu 1988, 79) geläufig zur Unterscheidung zwischen einzelsprachlicher Bedeutung und Sinn als ${ }^{\mathrm{C}}$ Bedeutung im Kontext' verwendet werden, mißverständlich vermengt werden. Diese Unklarheit ist bei der Analyse von Tempus und Aspekt besonders problematisch, weil hier eine genaue Differenzierung zwischen der Bedeutung der Form und deren Sinn in verschiedenen Kontexten essentiell ist. ${ }^{5}$

Diskutierenswert ist auch das Konzept des deiktischen Zentrums, das in bewußter Aussparung der zeitlichen Dimension als «Ich-hier-Zentrum» definiert wird [27]: «Es besteht keine Veranlassung, dem deiktischen Zentrum auf einer grundlegenden Beschreibungsebene eine implizite Eigenschaft des Typs [+jetzt] zuzubilligen. Das Konzept eines Jetzt setzt das Bewußtsein für ein Zeitintervall mit Namen Nicht-Jetzt voraus». Diese Aberkennung der Zeitdimension wirft jedoch einige offene Fragen auf: Gibt es in Sokols Augen kein Bewußtsein für das Nicht-Jetzt und soll folglich das Jetzt ausgespart werden? Woher nimmt sie die Gewißheit, daß es kein solches Bewußtsein für das Nicht-Jetzt gibt? Entscheidender ist aber, daß die Streichung der zeitlichen Dimension das Konzept der origo, die in den Einzelsprachen als Zentrum sprachlicher Teilsysteme wirkt, insgesamt entwertet. Das ego-hic-nunc als Quellpunkt der Rede ist ein unteilbares Ganzes, in dem jede Rede steht und das der Rede ihre Kontexte gibt (cf. Coseriu 1955-56, 46-51; Aschenberg 1999, 44-89): aus dieser elementaren Verankerung speist sich auch der Wert pragmatisch orientierter Untersuchungen, die etwa den Tempusgebrauch als Rede in komplexen Kontexten untersuchen.

Vor der Folie dieses semantischen Modells wird dann ein «Modell der verbalkategorialen Inhalte» [31-79] entworfen, das die aktionalen Muster über die Merkmale Dynamik, generelle Begrenztheit und Art der Begrenztheit (einseitig, zweiseitig) sowie über die phasische Strukturiertheit differenziert

${ }^{4} \mathrm{Zu}$ dieser Unterscheidung von Konzept und einzelsprachlicher Bedeutung cf. das Pentagon-Modell Raibles (1981, 5) und das Zeichenmodell von Blank (1997, 148). Cf. auch die vergleichende Besprechung dieser Zeichenmodelle und ihrer Traditionen bei Lebsanft/Gleßgen (2004, 12-17).

${ }^{5}$ In einer späteren Version des Schemas [29] ist allerdings nur noch von «Bedeutung» die Rede, cf. hierzu auch die Kritik von Werner (2001, 348s.). Die von Sokol intendierte Relation der Ebenen zueinander wirft ebenfalls Fragen auf [24]: «Die vier Ebenen stelle man sich im kommunikativen Prozeß korrelativ, die zwei Ebenen der Bedeutung interaktiv vor». Doch während im ersten Schema [23] die Relation der Ebenen zueinander durchweg als interaktiv $(\leftrightarrow)$ charakterisiert wird, findet sich im zweiten Schema [29] eine nicht näher begründete veränderte Setzung der Pfeile. Die graphischen Darstellungen können hier eine verbale Präzisierung der Begriffe und ihrer Relationen nicht ersetzen. 
[44s.]. Diese Merkmale sind nach Sokol für die Interaktion der Kategorien im Französischen relevant und können grammatische Restriktionen auslösen [45]. Grundlegend ist das Merkmal der Dynamik, das die nicht-dynamischen Stativa [46-48] von den anderen, dynamischen Mustern unterscheidet. Bezüglich der Begrenzungen sind nach Sokol nicht-begrenzte Stativa (Tout est calme), linksbegrenzte «L-Stativa» (Il reste) und beidseitig begrenzte «Interstativa» (Ma mère a sa migraine) zu unterscheiden. Bei den dynamischen aktionalen Mustern differenziert sie begrenzte und nicht-begrenzte Situationstypen. Nicht-begrenzt und dynamisch sind Progressiva (Ma mère travaille) und «Ph-Progressiva», die als zusätzliches Merkmal phasisch gegliedert sind und regelhaft wechselnde Subintervalle aufweisen [48s.]. Dynamisch und begrenzt dagegen sind «R-Progressiva» (Paul arrive), die ein Ziel als Rechtsgrenze aufweisen und traditionell als telisch bezeichnet werden [51s.], sowie die beidseitig begrenzten «Intergressiva» (Paul mange une pomme) [52s.]. Einen besonderen Fall stellen die Transitionen als «Schnittstelle zwischen zwei Ereignisräumen» [50s.] dar (Paul tire). Sokols Klassifizierung nutzt damit größtenteils Merkmale, die seit Vendler (1967) in der Beschreibung von Situationstypen Tradition haben, und arbeitet bei der Darstellung der aktionalen Klassen mit spatialisierenden Darstellungen, die zeitliche Konturen in räumlichen Bildern wiedergeben. Flankiert wird das so etablierte System der aktionalen Klassen durch die Definitionen von Aspektualität und Temporalität. Sokol faßt das temporal-deiktische Verweisen von Tempusformen als «explizite Temporalität» auf [62] und definiert futurische Temporalität knapp so, daß ein Futur vorliege, "wenn hinter der Aussage Es wird X geben steht Ich assertiere, es wird X geben» [68]. Sie folgt dem Konsens in der Aspektforschung, wenn sie Aspektualität ${ }^{6}$ als Perspektive auf eine Situation deutet und zwischen perfektivem und imperfektivem Aspekt dahingehend unterscheidet, daß beim imperfektiven Aspekt eine Innenperspektive, beim perfektiven Aspekt dagegen eine Außenperspektive gegeben sei [54s., 56].

Die zentrale Rolle von Aspekt und aktionalen Strukturen leitet Sokol daraus ab, daß Situationstypen «den Sonderstatus beanspruchen, direkt auf kognitive Verarbeitungsmuster schließen zu lassen» [35]. Diese kognitiven aktionalen Muster sollen anhand von Beispielsätzen im présent bzw. im Präsens (Paul rit, Paul hustet) erfaßt werden [49s.], die nach Sokol aufgrund der aspektuellen und temporalen Neutralität dieser Formen kognitive aktionale Strukturen ohne einzelsprachliche Modifizierung abbilden [40s.]. Zu hinterfragen ist hier zunächst die Prämisse der Neutralität des présent. ${ }^{7}$ So wird das présent als aspektuell neutral eingestuft, denn während imperfektive Formen

\footnotetext{
${ }^{6}$ Sokol bezeichnet die von sprachlichen Markierungen geleisteten aspektuellen Funktionen als «explizite Aspektualität» [54s.].

${ }^{7}$ Zur Diskussion um die aspektuelle Markierung des présent $\mathrm{cf}$. Schrott (1997a, 166-169). Auch Sokol [41] erwähnt die umstrittene aspektuelle Charakterisierung des présent.
} 
ausschließlich die «Involvierung» in eine Phase versprachlichen würden, könne das présent daneben gleichberechtigt den «natural endpoint» eines Sachverhalts versprachlichen [42]. Sokol spielt hier auf das «imperfective paradoxe» an, dem zufolge ein imperfektiver Aspekt bei telischen Verben die Zielerreichung stets ausblendet, wogegen aspektneutrale Formen beide Lesarten zulassen. Sieht man einmal davon ab, daß sich imperfektive Formen hinsichtlich des «imperfective paradoxe» keinesfalls homogen verhalten (Mitko 2000, 63s.), ergibt sich das Problem, daß das Konzept dieses «paradoxe» ein telischer Sachverhalt bestand, ohne sein Ziel zu erreichen - eine Retrospektive auf einen vergangenen Sachverhalt beinhaltet, die beim présent naturgemäß aber nicht gegeben ist. Daher sollte m. E. in der Diskussion um die Aspektmarkierung des présent nur bedingt mit dem (Nicht-)Erreichen des Telos argumentiert werden. Die temporale Unmarkiertheit des présent stützt Sokol vor allem auf die Schwierigkeit, ein «Jetzt des Sprechers/Sprechens» [43] zu definieren, das «wahlweise» als Zeitpunkt, Intervall oder Zeitstufe erscheine. Solche Defizite rein zeitreferentiell arbeitender Modelle können aber überwunden werden, wenn man erkennt, daß Tempora über die rein zeitliche Dimension hinaus Werte wie Aktualität und Verankerung im ego-hic-nunc ausdrücken (cf. Schrott 1997a, 129-136). Die angeführten Einwände zeigen, daß die Deutung des présent als neutrale Form nicht zwingend ist. Doch selbst wenn die postulierte aspektuell-temporale Neutralität des présent klarer erwiesen wäre, würde dies das Erkennen kognitiver aktionaler Muster nicht sicherstellen. Denn eine temporal-aspektuell unmarkierte Verbform hat ja auch einen semantischen Wert - und zwar den Wert einer Neutralität, der sich stets in Relation zu einem einzelsprachlichen System ergibt. Und dieser semantische Wert drückt dann dem außersprachlichen Sachverhalt im Akt der Versprachlichung ebenfalls seinen Stempel auf, so daß auch in diesem Fall keine Transparenz für kognitive Strukturen vorausgesetzt werden könnte.

Die Verwendung von Beispielsätzen im présent (bzw. im Präsens) bringt jedoch noch ein weiteres Problem mit sich. Beim Herausfiltern von aktionalen Strukturen aus Sätzen wie Paul rit geht Sokol implizit davon aus, daß diese Sätze eine aktionale Struktur gleichsam «in Reinform» ausdrücken. Diese Prämisse wird bei ihrer Kritik an dem in Schrott (1997a, 169) analysierten Beispiel Et puis d'abord tu m'énerves: je m'en vais deutlich [108]: «Die Einleitung des Satzes stellt außerdem eine Zusatzinformation dar, die man nicht ohne weiteres anfügen dürfte. Ein kontextloses Beispiel für ein typisch punktuelles achievement liegt in diesem Fall jedenfalls nicht vor». Entscheidend ist hier jedoch, daß auch beim Weglassen der «Einleitung» der Satz Je m'en vais kein kontextloses Beispiel darstellt. Denn Sätze wie Paul rit oder Je m'en vais sind zwar knapp und kontextarm, aber nicht kontextlos. Als Einheiten individueller Rede stehen sie stets in einem Redekontext, der als Verstehenskontext ihre Deutung implizit anleitet, ${ }^{8}$ und sind daher keine geeignete

${ }^{8}$ Zum Phänomen des vermeintlich kontextlosen Satzes cf. Meibauer (1986, 20-24). 
Basis, um das Funktionieren von aktionalen Strukturen im Zustand der Kontextlosigkeit zu untersuchen. ${ }^{9}$

Nach der kritischen Sichtung der Methodik der Beispielsätze soll nun auf den postulierten kognitiven Status der aktionalen Strukturen eingegangen werden. Sokol nimmt an, die Situationstypen seien «Muster, die von jenseits der Sprache kommend diese bis in formal-grammatische Bereiche hinein beeinflussen» und die stärker als andere Kategorien durch die Wahrnehmung determiniert seien [35]. Dieser Sonderstatus impliziere dann, wie eingangs erwähnt, daß Aspektualität und Aktionalität der Temporalität und der Modalität als Kategorien hierarchisch vorgeordnet seien [4]. Entscheidend ist hier natürlich die Begründung der kognitiven Natur der Situationstypen und die Zuverlässigkeit, mit der die in bestimmten «Verbprojektionen bzw. Sätzen» [44] versprachlichten Sachverhalte den Typen aktionaler Strukturen zugeordnet werden können. So wird die Einordnung von Paul rit als Ph-Progressiv durch die phasische Struktur des Lachvorgangs begründet, den Sokol als «〈Geräusch, Lachen〉 - 〈Pause〉 - 〈Geräusch, Lachen〉 usw.» beschreibt [49]. Da aktionale Strukturen in ihren Augen auf der kognitiven Ebene lokalisiert sind, ist diese Abfolge nicht einfach nur als intuitive Beschreibung gedacht, sondern will das kognitive Konzept LACHEN erfassen. Hier ist zu fragen, auf welcher Basis man dem Konzept LACHEN eine solche regelhaft-phasische Struktur zuweist, während das Konzept ARBEITEN (Ma mère travaille) als Progressiv eingeordnet wird, obwohl auch hier regelhaft wechselnde Subintervalle denkbar sind [48s.]. ${ }^{10}$ So fruchtbar die Arbeit mit eigenen Intuitionen sein kann, kognitive Konzepte von Sachverhalten - vor allem wenn sie eine so zentrale Rolle spielen - dürfen sich nicht allein aus dieser Quelle speisen. Die Zweifelsfälle der Zuordnung zeigen, daß die Bilder, die man mit Sachverhalten wie LACHEN oder ARBEITEN verbindet, auf persönlichen Erfahrungen und Assoziationen beruhen und sicher auch unterschiedlichen kulturellen Prägungen unterliegen können. Es erscheint daher sehr problematisch, wenn aus solchen intuitiven und subjektiven Bildern dann intersubjektiv gültige kognitive Strukturen herausgelesen werden. ${ }^{11}$

\footnotetext{
${ }^{9}$ Legt man solche kontextarmen Sätze Probanden vor, dann ergänzen diese einen Verstehenskontext, der allerdings implizit bleibt. Daher ist es transparenter, Beispiele mit einem reicheren Kontext zu verwenden, in dem die Kontextfaktoren explizit gegeben und damit in ihrem Wirken klarer beobachtbar sind.

${ }^{10}$ Ein ähnlicher Zweifelsfall findet sich [114, Tabelle] wo Il rit als Ph-Progressiv eingeordnet wird, während Il fume als Progressiv analysiert wird, obwohl man auch dem RAUCHEN eine regelhaft-phasische Struktur zubilligen kann.

${ }^{11}$ Cf. auch in einigen Fällen die ungenaue Abgrenzung zwischen sprachlicher und kognitiver Ebene: so wird die Kategorie der Aktionalität als «die semantische [Hervorhebung A. S.] Eigenschaft von Verbprojektionen bzw. Sätzen» bezeichnet [44], an anderer Stelle [46] ist von den «aktionalen Klassen des Französischen» die Rede. Wenn es sich jedoch um kognitive Strukturen handelt, dann können diese Strukturen nicht Klassen einer historischen Einzelsprache sein.
} 
Sprachwissenschaftliche Studien, die kognitiven Strukturen eine so große Bedeutung beimessen, sind in hohem Maße auf eine kognitionswissenschaftliche Absicherung angewiesen. Dabei gilt es auch zu präzisieren, wie sprachliche Strukturen und kognitive Konzepte und damit auch die Disziplinen Sprachwissenschaft und Kognitionswissenschaft bei spezifischen Fragestellungen ineinandergreifen. Sokol illustriert diese «Vernetzung» [35] am Beispiel des Konzeptes KOPF und dessen frames nach Detges ([1997, 5] 1999, 36s.). ${ }^{12}$ Die dort für den metonymischen Bedeutungswandel dokumentierte «Relevanz kognitiver Bildbereiche» [36s.] gelte auch für die Situationstypen, deren grundlegende Merkmale Durativität, Dynamik und Transitionalität [37-39] als Vorstellungen von Zeitlichkeit kognitiv auf räumlichen Bildern von Bewegung, Veränderung und Bildbegrenzung aufgebaut seien. Der knappe Verweis auf das Phänomen des metonymischen Bedeutungswandels ist allerdings kein Beleg für das Postulat, daß das entworfene Schema der Situationstypen kognitiven Strukturen entspreche. Der für den Primat der Interaktion von Aspekt und Aktionalität (gegenüber Temporalität und Modalität) zentrale kognitive Sonderstatus der Situationstypen bleibt eine Hypothese - nicht weniger, aber auch nicht mehr. Man könnte ebenso gut die Hypothese aufstellen, daß die Bilder der Situationstypen durch die Strukturen der zu untersuchenden historischen Einzelsprache geprägt sind. ${ }^{13}$

Eine (sprachwissenschaftliche) Argumentation mit kognitiven Strukturen erfordert eine solide kognitionswissenschaftliche Abstützung durch eine Erarbeitung des state of the art in den entsprechenden Disziplinen. Da ein solcher interdisziplinärer Dialog bei Sokol fehlt, sollten die Situationstypen nicht als kognitive Strukturen deklariert, sondern als ein sprachwissenschaftliches Modell genutzt werden, das seine Berechtigung dann aus seiner Erklärungskraft zieht und nicht aus einer in den Köpfen der Sprecher vermuteten Existenz. Die von Sokol [34] kritisierte Zurückhaltung der Pragmalinguistik gegenüber der Einbeziehung kognitiver Strukturen erscheint vor diesem Hintergrund klug und angemessen: die sprachwissenschaftliche Pragmatik konzentriert sich auf die Rede in Situationen und kann so den Schwerpunkt auf Hypothesen setzen, deren Überprüfung mit den genuinen Methoden der Sprachwissenschaft möglich ist. Die Idee, über für kognitive Strukturen transparente sprachliche Äußerungen zu kognitiven Mustern des vorsprachlichen bzw. außersprachlichen Denkens vorzudringen, ist originell, müßte aber sprachwissenschaftlich und kognitionswissenschaftlich weit stärker abgesichert werden.

\footnotetext{
12 Sokol bezieht sich auf eine frühere Fassung des Aufsatzes von Detges (1997, Paper zum Deutschen Romanistentag in Jena), die mit der 1999 publizierten Version weitestgehend übereinstimmt.

${ }^{13}$ So besteht etwa die Möglichkeit, daß der eine Klassifikation erarbeitende Sprachwissenschaftler sich dem Einfluß seiner Muttersprache bzw. der von ihm untersuchten Sprache(n) nicht entziehen kann und in diesen Einzelsprachen relevante Kriterien stärker berücksichtigt.
} 
Nach der Diskussion der aktionalen Klassen sollen die folgenden Abschnitte klären, inwiefern die Funktionsmuster der französischen Futura in Diachronie und Synchronie als Ergebnis einer Affinitäten und Restriktionen erzeugenden Interaktion von Aspekt und aktionalen Klassen deutbar sind.

\section{Futur simple und futur périphrastique: Ausgriffe in die Diachronie}

\subsection{Futur simple: Aspekt und aktionale Klassen in der Diachronie}

Nach Sokol ist das FS kein futurisches Tempus, sondern ein perfektiver Aspekt. Diese Hypothese ruht auf zwei Pfeilern: auf der diachronen Entwicklung des FS und auf dem synchronen Funktionsprofil des FS, die beide als Interaktionen von Aspekt und Aktionalität erklärbar seien. Damit soll die Entwicklung der cantare habeo-Periphrase als Interaktion des perfektiven Aspektes mit den verschiedenen aktionalen Klassen gedeutet werden. Auf der Basis eines schmalen Forschungsüberblicks ${ }^{14}$ [127-129] vertritt Sokol die Hypothese, daß die futurische Funktion des FS nicht aus dem modalen Wert der Obligationalität resultiere [128s.]. Sie beruft sich dabei hauptsächlich auf Benveniste (1968), der als Quellsemantik des FS die «Subordinations- und Futurwertigkeit einer 〈Infinitiv Passiv + habere>-Periphrase in Nebensätzen» angenommen und nicht im obligationellen Wert die Quelle der Periphrase gesehen habe [128].

Ein solcher nicht-modaler Ursprung der habere-Periphrase ist deshalb ein wichtiges Glied in Sokols Argumentation, weil die Entwicklung der Periphrase ja allein aus der Interaktion von Aspekt und aktionalen Klassen gedeutet werden soll und die Modalität in ihren Augen lediglich einen sekundären Effekt dieser Interaktion darstellt. Liest man die Darstellung bei Benveniste (1968, 89-91) nach, so ergibt sich ein differenzierteres Bild. ${ }^{15}$ Denn Benveniste unterscheidet zwischen ersten Verwendungen der Konstruktion im Nebensatz und einer späteren Ausweitung dieser Funktion, die dann in Konkurrenz zum synthetischen lateinischen Futur trete (1968, 89-91). Dabei hat die Periphrase nach Benveniste zwar keinen obligationellen Wert, doch schreibt er ihr durchaus eine modale Bedeutung zu, nämlich den Wert der «predestina-

\footnotetext{
${ }^{14}$ Bei der Darlegung der diachronen Entwicklung werden wichtige romanistische Studien nicht berücksichtigt. So bleiben etwa die Beiträge von Bodo Müller und Peter Wunderli und deren Kontroverse in der ZrP unerwähnt (Müller 1964, 1969, 1970 und Wunderli 1969, 1970a, 1970b); cf. auch die in diesen Beiträgen genannte Forschung zu den Futurperiphrasen.

${ }^{15}$ Eine detaillierte Diskussion ist schwierig, da Sokol ohne Angabe von Seiten auf Benveniste (1968) verweist. In der Fußnote zu Benveniste (n. 3 [127]) nennt sie lediglich drei weitere Studien - Fleischman (1982, 54), Bybee/Perkins/Pagliuca (1994, 187s.) und Lehmann (1995, 28s.) -, die ihrerseits auf den genannten Seiten auf Benveniste (1968) rekurrieren.
} 
tion» $(1968,90,91)$, der dann mit der von der synthetischen Form versprachlichten «intention» koexistiert habe. ${ }^{16}$

Wichtige Belege für die Annahme einer perfektiven und mit bestimmten aktionalen Klassen restringierten Periphrase sieht Sokol in übereinzelsprachlichen Tendenzen der Grammatikalisierung von habere-Fügungen [128s., cf. auch 201]. Eine solche Tendenz sei, daß haben-Periphrasen zuerst mit grenzbezogenen Ereignisprädikaten auftreten, sodann mit Progressiva, und erst spät oder überhaupt nicht mit Stativa kombinierbar seien [129]. Als «illustratives Muster» [131] für diese übereinzelsprachliche Tendenz wird zunächst die Grammatikalisierung des passé composé (PC) resümiert [131-133]. Die postulierten Restriktionen der cantare habeo-Periphrase für bestimmte aktionale Klassen werden aber weder durch spätlateinische oder altfranzösische Beispiele noch durch entsprechende romanistische Forschung untermauert. Vielmehr folgt eine Analyse der Restriktionen der «haben $z u+$ Infinitiv» Periphrase im heutigen Deutschen, die nach Sokol «mit der Quellkonstruktion des heutigen $f s$ [futur simple, A. S.] vergleichbar sein dürfte» [133] und die belegen soll, daß die cantare habeo-Periphrase ursprünglich keine modalobligationelle Bedeutung hatte, sondern daß das Zusammenwirken von Aspekt und Situationstyp vielmehr eine perfektiv-futurische Deutung auslöste, die dann sekundär Obligationalität ausdrücken konnte [137]. ${ }^{17}$ Diese Argumentation wirft die Frage nach der Tragfähigkeit der hier zugrundegelegten (und nicht näher begründeten) Analogie zwischen der deutschen haben zuPeriphrase und der romanischen cantare habeo-Periphrase auf. Das Deutsche der Gegenwart sowie die früheren Sprachzustände des Französischen stellen unterschiedliche einzelsprachliche Systeme dar, die auch durch verschieden gestaltete Tempus- und Aspektsysteme charakterisiert sind, so daß die Berechtigung solcher Analogieschlüsse auf der Basis weniger Belege [135-137] bezweifelt werden darf.

Neben dem Rekurs auf allgemeine und übereinzelsprachliche Regeln führt Sokol auch einen «sprachgeschichtlichen Beleg» für die Restriktion gegenüber Stativa an [137s.]. So sei die Periphrase, als sie bereits «durchgängig perfektiv-futurisch» verwendet wurde [137], noch nicht generell auf Stativa ausgedehnt gewesen: «Die futurische Funktion bei Stativa mit der Kopula sein deckte im 11. Jahrhundert immer noch die aus dem Latein tradierte Futurform ab (lat. ero $\rightarrow$ afrz. er, ier)» ([138], mit Verweis auf Lausberg ${ }^{2} 1972,226$ s. in

16 Auf der Basis von Benveniste (1968) schließen Bybee/Pagliuca/Perkins (1994, 261-263), daß Konstruktionen mit BE und HAVE die Bedeutung «predestination» haben und sich zu Formen mit der Bedeutung «prediction» entwickeln, wobei sie nicht notwendig durch das Stadium der «obligation» gehen müßten - dieser Spur wäre in der Diskussion um modale Werte der cantare habeo-Periphrase natürlich ebenfalls nachzugehen.

17 Während deutsche Beispiele belegen sollen, daß Stativa nicht perfektiv-futurisch lesbar seien [135s.], wird ein englisches Beispiel als Beleg dafür angeführt, daß Stativa in haben-Periphrasen überwiegend modal gedeutet werden [137]. 
n. 18 [ibid.]). Diese alte Futurform finde sich in allen grammatischen Personen mit Ausnahme der 2. Person Plural als der üblichen Anredeform ([138], mit Verweis auf Bonnard/Régnier 1991, 117 und Raynaud de Lage ${ }^{12} 1975,97$ in n. 19 [ibid.]). Aus dem Fehlen von Belegen des alten Futurs für die 2. Person Plural schließt Sokol nun, daß «Anreden in der 2. Person diejenige syntaktische Umgebung darstellten, in der das neue Futur für die Prädikate mit seinKopula zuerst obligatorisch wurde» [138]. Demnach hätten die Stativa Eingang in das perfektive FS über den Umweg der 2. Person Plural gefunden, wo sie durch die Imperativität nicht statisch wirkten und über diese «Sonderfunktion» [ibid.] in das FS eingeschleust werden konnten.

Diese abstrakte Argumentation, die auf Textbelege verzichtet, enthält allerdings mehrere Klippen. Denn bei Lausberg $\left({ }^{2} 1972,226 \mathrm{~s}\right.$.) findet sich lediglich der kurze Hinweis, daß «das lat. Futurum (außer lat. ero: § 918)» im Romanischen nicht weiterlebe. Der genannte Paragraph (Lausberg ${ }^{2} 1972,268$ ) enthält dann den Vermerk: «Das alte lat. Futur ero ist als Futur noch erhalten in afr. ier, apr. er». Wenn Sokol mit Verweis auf Lausberg den Schluß zieht, daß die Periphrase nicht auf «Stativa mit der Kopula sein» ausgedehnt gewesen sei, so daß das alte Futur die Lücke habe füllen müssen [138], dann geht diese Deutung weit über die knappen Informationen bei Lausberg hinaus. Ferner stellt sich die Frage nach der Datenbasis des angeführten «sprachgeschichtlichen Belegs». Denn wie schwer das von Sokol angeführte Argument des Fehlens - genauer: des Nicht-Belegtseins - einer Form wiegt, hängt natürlich vom Umfang des gesichteten Korpus ab. Die Feststellung, die alte Futurform finde sich «in altfranzösischen Texten [Hervorhebung A. S.]» [138] in allen grammatischen Personen außer in der 2. Person Plural, fußt, wie bereits erwähnt, auf zwei Lehrwerken zum Altfranzösischen. Hier findet sich bei Raynaud de Lage $\left({ }^{12} 1975,97\right)$ eine Konjugationstabelle zu estre mit dem Vermerk, daß die 2. Person Plural nicht belegt sei, und Bonnard/Regnier (1991, 117) geben eine Auflistung der estre-Formen ohne die 2. Person Plural («paradigme défectif»). Die zitierte Forschung liefert damit zwar anregende Ausgangspunkte für Sokols Hypothese, doch sie stellt eine zu schmale Basis dar, als daß man von einem «sprachgeschichtlichen Beleg» für die Restriktion gegenüber Stativa sprechen könnte. ${ }^{18}$ Entscheidender ist jedoch, daß die (möglicherweise) gegebene Affinität der habere-Periphrase zur 2. Person auch ganz anders gedeutet werden könnte, und zwar gerade als Beleg für den modalen Gehalt der Periphrase. Denn Obligationen haben eine natürliche Wahlverwandtschaft zur 2. Person als Form des Appells an andere, so daß die Affinität zwischen 2. Person und habere-Periphrase vielmehr als Indiz für eine modalobligationelle Semantik gewertet werden könnte. ${ }^{19}$

\footnotetext{
${ }^{18}$ Als ökonomische Alternative zur natürlich sehr aufwendigen Analyse eines altfranzösischen Korpus hätte sich z. B. die Suche nach entsprechenden Belegen im Tobler/Lommatzsch angeboten.

${ }^{19}$ Dieses Argument wird übrigens auch durch den oft zitierten Futur-Beleg aus der Fredegar-Chronik gestützt. In dem Dialog - «ille respondebat «non dabo», Justinianus
} 
Ein weiteres wichtiges Argument für die Hypothese eines aktional restringierten FS liefern nach Sokol übereinzelsprachliche Charakteristika der Infinitiv-Form [139s.]. So gehe das prospektivierend-futurische Element des FS auf eine Bedeutungskomponente des Infinitivs zurück [140s.]. Diese Qualität des Infinitivs verknüpft Sokol mit dem Verhalten von Infinitiv-Reihen des Typs «Inf., Inf., Inf., quel bonheur!» im heutigen Französisch [140s.], wo eine solche Reihe nur mit begrenzt-dynamischen Prädikatklassen Sequentialität ausdrücken könne [Beispiele ibid.]. Aus beiden Charakteristika schließt Sokol [141], daß «bei nicht-begrenzten und statischen Prädikaten dieses prospektivierende Element diachron entfiel». Daher habe die Form in Kombination mit Stativa nie als Futur fungieren können [ibid.]: «Die Gesamtkonstruktion wäre damit mit bestimmten Klassen nie ein 〈echtes〉, selbständiges Futur gewesen und ist es vermutlich auch heute nicht». Nach Sokol sind die Intergressiva beim FS die diachron älteste aktionale Klasse [146], und die rechtsbegrenzten «R-Progressiva» werden ebenfalls als «diachron früh» eingestuft [150]. Diese Aussagen werden allerdings weder durch Belege aus früheren Sprachzuständen noch durch Verweise auf ein diachrones Korpus untermauert, und auch die Ausführungen zum Infinitiv sind nicht völlig stichhaltig. ${ }^{20}$ Denn abgesehen davon, daß der Ausdruck zeitlicher Sukzession in Infinitivreihen neben der Aktionalität auch entscheidend davon abhängt, ob die Sachverhalte nach unserem Weltwissen eher simultan oder sukzessiv auftreten, stellen die freien Infinitiv-Reihungen und die Verbalperiphrase doch deutlich unterschiedene Fälle der Infinitivverwendung dar, so daß der Analogieschluß zwischen beiden fraglich erscheint.

Die kritische Sichtung zeigt, daß Sokol anregende Hypothesen zur Entwicklung des FS formuliert. Doch wenn sie in ihrer «Zwischenbilanz» resümiert, man habe für $P C$ und $F S$ «diachron in analoger Weise vorgeführt, wie sich entlang der semantischen Leiste, die die aktionale Struktur bietet, im Zuge der Ausdehnung auf vormals nicht zulässige Prädikate der kommunikative Schwerpunkt verlagern kann» [144], dann beinhaltet diese Feststellung schlichtweg eine Fehleinschätzung der angeführten Argumente. Denn die Entwicklung des FS wurde eben nicht «diachron in analoger Weise vorgeführt» [ibid., Hervorhebung A. S.]. Vielmehr wurde aus synchronen Befunden verschiedener einzelsprachlicher Systeme zusammen mit übereinzelsprachlichen Tendenzen der Grammatikalisierung und atomistischen Ausgriffen in die Diachronie auf eine solche diachrone Entwicklung geschlossen, ohne diese selbst zu untersuchen. Im Kontext oft skizzenhaft knapper Darstellungen der diachronen Entwicklungen, wie Sokol sie unternimmt, ist es natürlich nicht mög-

dicebat 〈daras»» (zit. nach Müller 1964, 76) - wird auf die im «Normalfutur» (Müller ibid.) versprachlichte Weigerung Non dabo hin vom Angesprochenen diejenige FuturForm gewählt, die die Zukunft als Obligation versprachlicht, nämlich Daras im Sinne von 'Du hast doch zu geben'.

${ }^{20}$ Die fundierte Studie Wunderlis, die ausführlich auf die Infinitiv-Form eingeht (Wunderli 1969, bes. 395-398), wird nicht berücksichtigt. 
lich, die komplexe Entwicklung der spätlateinischen bzw. frühromanischen Futurperiphrasen abzuhandeln, ${ }^{21}$ und es erscheint auch völlig nachvollziehbar, daß nicht auf ein eigenes diachrones Korpus zurückgegriffen wird. ${ }^{22} \mathrm{Al}-$ lerdings müßte dann in der Argumentation für ein perfektives FS das Gewicht solcher Exkurse auch adäquat eingeschätzt werden. Das Postulat, das FS habe «zu keinem Zeitpunkt seines grammatischen Werdegangs futurischen Wert unabhängig von aktionalen Klassen gehabt» und sei «strenggenommen gar kein Tempus» [145], bleibt daher hypothetisch, und auch die später getroffene Feststellung, «Offensichtlich hat sich das Funktionsspektrum seit dem Altfranzösischen kaum verändert» [167], ist sprachgeschichtlich nicht ausreichend untermauert.

\subsection{Futur périphrastique: aller-Periphrase und aktionale Klassen}

In ihrem Überblick zur Grammatikalisierung des FP [169-175] vertritt Sokol die Position, daß die aller-Periphrase schon im Altfranzösischen implizit futurisch fungierte [172] und dabei als «expliziter Perfektiv-Marker (bei bestimmten Prädikaten)» gewirkt habe [173]. Aufgrund der Zielort-Semantik sei die Infinitiv-Fügung an grenzbezogen-dynamische Prädikate gebunden [178]. So zeigen nach Sokol [ibid.] «[d]ie Beispielsätze aus der Sprachgeschichte, die in der Forschungsliteratur zu finden sind» die Periphrase «ausschließlich kombiniert mit Intergressiva, R-Progressiva sowie mit Transitionen, die durch ein agentives Subjekt zum R-Progressiv verschoben sind». ${ }^{23}$ In der Forschungsliteratur - Sokol nennt hier Gougenheim (1929), Brüser (1989 [unveröffentlichte Magisterarbeit]) und Detges ([1997] 1999) - fände sich kein Beispiel mit einem «Progressiv oder Stativ (sei es unbegrenzt oder begrenzt)» [178]. Allerdings seien «die Verben savoir, connaître und voir» [ibid.] belegt, was damit erklärt wird, «daß die Prädikate, in denen sie erscheinen, tatsächlich eher als L-Stativa zu betrachten wären, was ihnen als u. a. transitionelle Prädikate bereits früh Zugang zur Periphrase verschaffte». Die einzelnen Beispiele, die solche Umdeutungen verdeutlichen könnten, werden jedoch nicht angeführt. Auf diesen Befunden aufbauend, schließt Sokol auf eine Restriktion des FP auf grenzbezogene und dynamische Prädikate, deren Gültigkeit für das Neufranzösische zu überprüfen sei [178].

\footnotetext{
${ }^{21}$ Um die Entwicklung des Typus cantare habeo präzise darzustellen, müßten z. B. neben der lateinischen synthetischen Form auch andere Versprachlichungsmöglichkeiten des Spätlateinischen und der frühen romanischen Sprachen berücksichtigt werden (cf. Müller 1964, 54). Die Fokussierung auf cantare habeo entspricht einer gegenwartszentrierten Perspektive, die die Dynamik des Sprachwandels nicht völlig erfassen kann.

${ }^{22}$ Cf. etwa Sokol (n. 4 [127]): «Zur Theorie über den Infinitiv Passiv kann ich mich mangels direkter Zugriffsmöglichkeit auf die lateinischen Quellen nicht äußern».

${ }^{23}$ Diese diachrone Entwicklung der Form mit ihrer Ausdehnung auf zunächst nicht selegierte aktionale Strukturen bilde sich, so Sokol, in den synchronen Funktionsmustern des Gegenwartsfranzösischen ab [174, 177, 192].
} 
Wie stichhaltig ist dieser Exkurs in die Diachronie? Das Postulat einer Bindung der aller-Periphrase an grenzbezogen-dynamische Prädikate wird durch den Verweis auf Beispielsätze in der Forschungsliteratur gestützt. Der genannte Beitrag von Ulrich Detges ([1997] 1999) geht kurz auf die Interaktion von aller-Periphrase und aktionalen Klassen ein, und zwar verweist er interessanterweise auf ein Merkmal, das Sokol sowohl in ihrer Systematik der aktionalen Klassen [51, 53] als auch bei ihrem diachronen Aufriß zum FP ausklammert - nämlich das Merkmal der Agentivität. So ist nach Detges ([1997, 9] 1999,41 ) bei (s'en) aller faire die diachronische Entwicklung von der Bedeutung «die Absicht haben, etwas zu tun» zur Bedeutung «Zukunft» dadurch charakterisiert, daß zunehmend Verben verwendet werden, die Handlungen versprachlichen, die der Träger der Handlungsabsicht nicht kontrolliert. Für die Zugänglichkeit von Verben zur aller-Periphrase könnte daher die Agentivität eine wichtige Rolle spielen. In den Ausführungen Gougenheims (1929) zur aller-Periphrase (1929, 83-84, 92-113) wird die Interaktion von aktionalen Strukturen und Aspekt dagegen nicht thematisiert. ${ }^{24}$ Als Datengrundlage für eine Hypothese zur Interaktion von aller-Periphrase und Aktionalität sind die Beispiele beider Studien nicht geeignet, denn dieses Korpus ist nicht nur relativ klein, es stammt auch aus Untersuchungen mit anderen Forschungszielen. Aufgrund der mangelnden Transparenz der Angaben zu den Belegen aus der Forschungsliteratur - Beispiele für die postulierten Affinitäten, Umdeutungen und Restriktionen [178] werden weder aufgeführt noch diskutiert - ist ein vertieftes Eingehen auf Sokols Argumentation nicht möglich und würde eine eigene materialbasierte Studie erfordern. Wie schon bei der diachronen Darstellung des FS sind auch die Postulate zur diachronen Entwicklung des FP und zu möglichen Restriktionen korpuslinguistisch nicht gesichert und können lediglich den Status von Hypothesen beanspruchen.

\section{Futur simple und futur périphrastique: synchrone Funktionsmuster}

Da Monika Sokol, wie schon erwähnt, ihre Darstellung der Futura im heutigen Französisch eng an meine Studie anbindet, soll einleitend das in Schrott (1997a) dargelegte «Prinzip der Konditionierung» vorgestellt werden, bevor kontrastiv das von ihr entworfene Modell der Interaktion von Aspekt und Aktionalität rezipiert wird. Um die Erklärungskraft beider Modelle für die Konkurrenz von FS und FP zu vergleichen, wird die bei Sokol getrennte Abhandlung der beiden Formen in kontrastiven Analysen gebündelt, wie sie in Schrott (1997a, 1997b) systematisch durchgeführt werden. Die in solchen Analysen dokumentierten Restriktionen beider Formen bilden dann einen Prüfstein für beide Modelle.

${ }^{24}$ Sichtet man etwa die Beispiele bei Gougenheim, dann finden sich auch nichtgrenzbezogene Verben, cf. z. B. Gougenheim (1929, 99): «Je voys chevaucher ung baston / En deffaulte d'une jument». 


\subsection{Futur simple vs. futur périphrastique: das «Prinzip der Konditionierung»}

In Schrott (1997a) - einer gezielt auf das Französische der Gegenwart konzentrierten Studie - wird die Opposition von FS und FP als eng verzahnte Konkurrenz analysiert, die auf dem Prinzip einer aktuellen oder virtuellen Konditionierung beruht. Dieses Prinzip ist eine Präzisierung der in der Forschung übereinstimmend beschriebenen Differenzierung zwischen Kontinuität und Zäsur zur Sprechsituation (cf. den Forschungsbericht bei Schrott 1997a, 26 -40): während das FP Zukünftiges in Kontinuität zur Sprechsituation versprachlicht, drückt das FS eine Zäsur zum ego-hic-nunc aus.

Auf der Basis einer umfangreichen, mit Kommutationen und Sprecherbefragungen arbeitenden Korpusauswertung (cf. zur Methodik Schrott 1997a, 1215) kann diese Unterscheidung induktiv bestätigt und verfeinert werden. Demnach versprachlicht das FP teilaktuelle Sachverhalte, bei denen die Bedingungen für die Realisierung bereits erfüllt sind, so daß eine aktuelle Konditionierung vorliegt. Da der futurische Sachverhalt in einer Vorstufe bereits gegeben ist, liegt eine Teil-Ganzes-Beziehung vor (Schrott 1997a, 54). Diese meronymische Semantik manifestiert sich oft als Ursache-Wirkung-Relation und kann als prospektiver Aspekt beschrieben werden (Schrott 1997a, 107109). Dagegen hängen Sachverhalte im $F S$ von noch unerfüllten Bedingungen $\mathrm{ab}$, sind damit an eine virtuelle conditio gebunden und haben in diesem Sinne virtuelle Semantik (Schrott 1997a, bes. 30-32; 1997b, 41-42). Auch wenn Sachverhalte im FP ganz überwiegend eine in der Sprechsituation erfüllte Bedingung ausdrücken, finden sich Beispiele, in denen die erfüllte conditio in einer Referenzsituation gegeben ist, die nicht mit der Sprechsituation zusammenfällt, sondern in der Zukunft lokalisiert ist. Entscheidend ist nun, daß auch bei diesen Verwendungen, die eine Ausweitung des Gebrauchs darstellen könnten, die meronymische Semantik des FP erhalten bleibt (cf. Schrott 1997a, 55s.). Das FP profiliert sich damit als Futurform, die Zukünftiges als im Werden begriffen darstellt, während das FS eine Existenz in der Zukunft ausdrückt. Diese Opposition bedingt nun eine unterschiedliche Art und Weise der Lokalisierung in der Zukunft (cf. Schrott 1997a, 57-67 und 1997b, 42s.). ${ }^{25}$ Während das FS lediglich die Aussage trifft, daß ein Sachverhalt zu einem nicht näher spezifizierten Zeitpunkt in der Zukunft existent sein wird, beinhaltet das FP, daß der teilaktuelle Sachverhalt, der auf dem Weg der Zieleinlösung ist, zu einem spezifischen Zeitpunkt in der Zukunft (der allerdings nicht explizit gegeben sein muß) realisiert wird und in diesem Sinne «Orientiertheit» besitzt. Die Opposition von aktueller und virtueller Konditionierung illustriert das folgende Beispiel: ${ }^{26}$

\footnotetext{
25 Ein weiterer Ausdruck der unterschiedlichen Konditionierung ist die Opposition von Ulteriorität und Kontiguität; cf. Schrott (1997a, 41-57 und 1997b, 42-43).

${ }^{26}$ Beispiel aus: Nouvel Observateur 1574, 44; zit. nach Schrott (1997a, 32). In den Textbeispielen ist die Verbform des Originaltextes jeweils kursiv wiedergegeben.
} 
(1) En 1992, tandis que Nicole Brown Simpson entame une procédure de divorce, son mari la harcèle, l'espionne et tente de s'introduire chez elle. En 1993, il défonce sa porte à coups de pied et la menace en vociférant. Épouvantée, Nicole appelle la police: «Il (va me tuer - *me tuera)!»

Die von der Sprecherin befürchtete Gewalttat kündigt sich bereits im unbeherrschten Toben des Ehemannes an und ist damit aktuell konditioniert. Das FS würde dagegen versprachlichen, daß die Gewalttat von einer unerfüllten Bedingung abhängt, rein virtueller Natur ist und «irgendwann» in der Zukunft eintreten wird - eine Lesart, die im vorliegenden Kontext ausscheidet. Im Unterschied zu (1) sind im nächsten Beispiel sowohl FS als auch FP möglich, allerdings mit unterschiedlichen Nuancen: ${ }^{27}$

(2) «Joseph, dit soudain ma mère toute pâle, j'ai un pressentiment!» Mon père se mit à rire: «Moi aussi, dit-il. J'ai le pressentiment que (nous allons passer - nous passerons) des vacances magnifiques! J'ai le pressentiment que (nous allons manger - nous mangerons) de belles brochettes de grives, de darnagas et de perdrix! J'ai le pressentiment que les enfants (vont prendre - prendront) trois kilos chacun!»

Während das teilaktuelle $F P$ die guten Vorahnungen des Sprechers fokussiert und ausdrückt, daß die Ferienfreuden gleichsam schon begonnen haben, würde das FS die positiven Wirkungen der Ferien als virtuelle Sachverhalte versprachlichen. Da die vom FS kontrastiv zum FP des Originals geleistete Nuance in diesem Kontext ebenfalls eine sinnvolle Lesart darstellt, sind beide Formen möglich.

Die so erarbeiteten Tempusprofile erklären nicht nur die Verwendungen von $F S$ und FP in Tempussequenzen, das Zusammenspiel von Futursemantik und Aktanten und die Verwendung der Futura in Konditionalsatzgefügen, sondern auch die unterschiedlichen pragmatischen Potentiale von FS und FP (Schrott 1997a, 72-140). Sokols ganz auf die Interaktion von Aspekt und aktionalen Klassen zentrierte Arbeit will nun belegen, daß das in Schrott (1997a) dargelegte «Prinzip der Konditionierung» lediglich ein Epiphänomen der Interaktion von Aspekt und aktionalen Klassen sei.

\subsection{Futur simple im Französischen der Gegenwart}

Nach Sokol ist das FS ein perfektiver Aspekt, der in Interaktionen mit verschiedenen aktionalen Strukturen temporale und modale Werte als synchrone Funktionsmuster erzeugt [146-164]. So werde bei Transitionen die perfektive Komponente der Prädikate noch zusätzlich durch das perfektive $F S$ verstärkt [153], wogegen die perfektive Außenperspektive bei nicht-grenzbezogenen Prädikaten erst eine begrenzende Gesamtkontur erzeuge [150]. Bei den Progressiva

${ }^{27}$ Beispiel aus: Pagnol, Marcel, Le château de ma mère. Souvenirs d'enfance, Paris, Éditions de Fallois, [1957] 1988, 179; zit. nach Schrott 1997a, 132s. 
löse der perfektive Aspekt primär eine iterative Lesart aus, doch seien futurische Werte ebenfalls möglich [151s.]. ${ }^{28}$ Bei Progressiva mit Tendenz zu intergressiver Lesart leiste der perfektive Aspekt des FS zudem eine Desambiguierung. Während im (nach Sokol) neutralen présent ein Satz wie X mange la soupe perfektiv («X ißt die Suppe [ganz] auf») oder imperfektiv («X ißt Suppe») deutbar sei [151], könne der Satz $X$ mangera la soupe dank des perfektiven Aspekts des FS nur die perfektive Lesart «X ißt die Suppe (demnächst ganz) auf» haben. Dieser Effekt soll an einer kleinen Beispielreihe überprüft werden:

(3) Il mangera la soupe.

(4) Il mangera sa soupe.

(5) Il mangera de la soupe.

Die Sprecherbefragung bestätigt die Festlegung der Lesart durch das perfektive FS nicht. So kann die Suppe in (3) und (4) ganz oder teilweise verzehrt werden, je nach Verstehenskontext. Und in (5) ist trotz des FS durch den gewählten article partitif sogar festgelegt, daß eine unbestimmte Menge Suppe gegessen wird. Würde das FS aber kraft seines Aspektes eine perfektive Lesart festlegen, dann müßte diese Lesart in jedem Fall gegeben sein und dürfte nicht wie in (5) durch einen veränderten Artikel ausgehebelt werden.

Das markanteste Muster der Interaktion wird jedoch für die Stativa postuliert, bei denen zwischen unbegrenzten Stativa und Stativa mit Grenzbezogenheit (L-Stativa, Interstativa) zu unterscheiden sei. Grundsätzlich könnten grenzbezogene Stativa (wie savoir) im FS vorkommen und würden dann meist transitionell reinterpretiert, so daß epistemisch-modale Werte eher selten seien $[156,158]$. Der Stativa-Wert in temporaler Lesart bleibe nur erhalten, wenn die Futurität durch den Kontext gewährleistet sei [156]. Beispielanalysen (Schrott 1997a, 113-114) und (darauf aufbauende erneute) Sprecherbefragungen legen jedoch nahe, daß der Redekontext für die Deutung solcher grenzbezogener Stativa des Typs savoir eine entscheidende Rolle spielt. Nach Sokols Schema würde ein Satz wie Il saura la vérité in den meisten Fällen transitionell umgedeutet werden ('neues Wissen erwerben'), könnte aber auch modal reinterpretiert werden; eine temporale Deutung als Stativ ('über Wissen verfügen') wäre jedoch aufgrund der fehlenden futurischen Verankerung nicht möglich. Untersucht man Verwendungen von savoir im FS in konkreten Beispielen, dann erkennt man, daß es ganz entscheidend ist, ob der Kontext einen künftigen Neuerwerb oder aber ein künftiges Verfügen über Wissen nahelegt (cf. Schrott 1997a, 113s.). So kann in temporaler Lesart etwa die Äußerung Il saura la vérité sowohl transitionell als auch statisch aufgefaßt werden. Und auch bei futurischer Situierung ist die Deutung als Stativ nicht die

28 Die Annahme einer primär iterativen Lesart bei Progressiva erscheint aber nicht zwingend. So kann Quand je chanterai tu pleureras [152] gleichermaßen singulär und iterativ gelesen werden. Ein Beispiel wie Quand Pierre chantera tu pleureras belegt, daß Sokols Annahme, die nicht-iterative Lesart sei aufgrund der 1. Person möglich, nicht zutrifft. 
einzig mögliche; so kann in Quand il arrivera demain, il saura la vérité der Sachverhalt je nach Kontext als 'Erfahren der Wahrheit' oder als 'Wissen um die Wahrheit' verstanden werden. Ein solcher Kontexteinfluß spricht jedoch gegen einen Automatismus von Aspekt und aktionaler Klasse, der ein kontextunabhängiges, festes Interaktionsmuster aufweisen müßte.

Den strengsten Restriktionen unterliegen nach Sokol beim FS aber die unbegrenzten Stativa, die im FS mit temporalem Wert nur möglich seien, wenn der Sachverhalt begrenzt gedacht oder dynamisch-iterativ umgedeutet werden könne [158s.] oder wenn eine temporal-futurische Angabe gegeben sei [160]. Dank solcher Umdeutungen seien Sätze wie

(6) Ils seront tous malades.

(7) On ne sera jamais assez sévère avec les voleurs.

mit temporalem Wert möglich [158s.]. So sei (6) futurisch-transitionell lesbar («Irgendwann wird ihnen allen schlecht») ${ }^{29}$ und (7) sei iterativ deutbar («Man kann mit Dieben gar nicht streng genug verfahren»). Doch während in (6) der Sachverhalt «être malade» in der Tat als begrenzter Zustand auffaßbar ist, muß «être sévère avec les voleurs» (7) nicht zwingend dynamisch-iterierend verstanden werden, sondern kann auch Ausdruck einer kontinuierlich gegebenen Haltung sein.

Wichtig ist nun, daß es nach Sokol Stativa gibt, denen auch der Weg über transitionelle oder modale Umdeutungen versperrt sei. Solche Stativa seien im perfektiven $F S$ «nicht akzeptabel» und seien «ohne Kotext im fs [futur simple, A. S.] nicht sinnvoll zu dekodieren» [160]: ${ }^{30}$

(8) *New York sera une très belle ville.

(9) *Ma tante, ce sera une femme blonde.

Wenn die Restriktion dieser Stativa durch das FS als grammatischer perfektiver Aspekt bedingt ist, dann müßte diese Restriktion als Teil des einzelsprachlichen Systems auch in einem veränderten Kontext Bestand haben. Dieser Einschätzung widerspricht jedoch eine französischen Sprechern vorgelegte Beispielreihe mit den fraglichen Stativa, aus denen zwei Belege herausgegriffen werden sollen:

(10) Berlin sera une très belle ville.

(11) Norma Jean a pris sa décision: elle sera une femme blonde, elle sera belle.

Beispiel (10) wäre etwa möglich als Äußerung eines Besuchers auf der «Baustelle der Republik», und Beispiel (11) ist denkbar im Kontext einer Frau, die beschließt, zukünftig eine blonde Schönheit zu sein. Der (denkbare) Einwand,

\footnotetext{
29 Allerdings wäre als Paraphrase m. E. eher «Sie werden alle krank sein/werden» anzusetzen.

${ }^{30}$ Beispiel (8) stammt aus Schrott (1997a, 305) und wird bei mir als Ausdruck einer Vermutung kontrastiv zur devoir-Periphase getestet, wobei das FS in diesem Fall in der Funktion futur de probabilité restringiert ist.
} 
lediglich der veränderte Kontext mache die Beispielsätze (10) und (11) akzeptabel, ist dabei nicht stichhaltig, da er die Ebenen der Sprachkompetenz im Sinne Coserius (1988, 76-89) vermengt. Denn das Postulat der Nicht-Akzeptabilität dieser Stativa beim FS gehört der Ebene des einzelsprachlichen Systems an, während das Verstehen konkreter Beispiele auf der Ebene der Texte argumentiert. Wenn Sokol [160] erklärt, bestimmte Stativa seien im FS nicht akzeptabel und die Beispiele nicht sinnvoll dekodierbar, dann sind das zwei Postulate, die auf verschiedenen Ebenen des Sprechens lokalisiert sind. In Erinnerung zu rufen ist auch, daß die bei Sokol [ibid.] angeführten Beispiele ebenfalls keine kontextlosen Sätze darstellen, sondern als Rede stets einen Redekontext haben, in dem das FS dann akzeptabel oder inakzeptabel bzw. sinnvoll oder sinnschwach ist.

Die Beispieldiskussion hat gezeigt, daß die postulierten Restriktionen bei weitem nicht so rigide sind, wie Sokol annimmt. Dazu kommt, daß die präsentierten Einschränkungen nur für $F S$-Verwendungen ohne futurisch situierenden Kontext gelten, denn in Kontexten mit futurischer Referenz kann das FS auch im Rahmen von Sokols Modell mit allen aktionalen Klassen - einschließlich der unbegrenzten Stativa [160] - futurisch fungieren [156, 160, 202]. Auch die Daten der Korpusauswertung stützen die Strenge der Restriktionen nicht, hat doch ausgerechnet das angeblich bei nicht-grenzbezogenen Prädikaten restringierte FS bei unbegrenzten Prädikaten den höchsten Anteil. ${ }^{31}$ Sokol erklärt dieses Ergebnis damit, daß sämtlich Reinterpretationen (transitionell oder modal) vorliegen würden und nur ein einziges futurisch fungierendes unbegrenztes Stativ gegeben sei [167]. Doch da bei der Datenauswertung kein einziges Beispiel für eine solche Reinterpretation angeführt wird und auch der erwähnte einzige Beleg [ibid.] für ein futurisch fungierendes Stativ weder zitiert noch durch Quellenverweis zugänglich gemacht wird, erlaubt die Korpusdarstellung dem Leser hier keine weitere Bewertung und Diskussion dieser Aussagen. ${ }^{32}$

\subsection{Futur périphrastique im Französischen der Gegenwart}

Bei den synchronen Funktionsmustern des FP [184-192] werden bei Sokol neben «lexikalischer Konkretverwendung» und «semilexikalischer Verwendung» - eine semigrammatische Verwendung, eine prospektiv-inchoative Verwendung und eine temporal-futurische Verwendung angeführt. Die semigram-

\footnotetext{
31 Sokols Datenanalyse zu den aktionalen Klassen beim FS [167] kommt zu dem Resultat: «Der Anteil unbegrenzter Prädikate beträgt insgesamt 27,29\% gegenüber $13,63 \%$ pf [présent futural, A. S.] und 16,52\% fp [futur périphrastique, A. S.])». Bei den «selbständig fungierenden Daten» ergebe sich ein verändertes Bild: «Dort sinkt der Prozentsatz aller Stativa gegenüber der Gesamtstatistik auf 18,90\% (gegenüber $13,75 \% f p$ und [...] 9,28\% im $p f »$. Allerdings bleibt auch in letzterem Fall das $F S$ die häufigste Form bei den Stativa.

32 So kann nicht beurteilt werden, ob diese Daten tatsächlich einen «wichtigen Beleg für die Hypothese, daß das sog. fs [futur simple, A. S.] primär ein perfektives Signal ist» liefern [167, cf. auch 203].
} 
matische Verwendung, die eine «Geneigtheit bzw. Intention des Subjektreferenten» ausdrücke [185], signalisiert nach Sokol bezogen auf den Subjektreferenten primär präsentische Imperfektivität und «sekundär eine perfektive Außenperspektive auf ein bereits futurisch verlagertes Ereignis E» [186], so daß hier imperfektive und perfektive Züge verbunden zu sein scheinen. Bei der semigrammatischen Periphrase, deren Nutzwerte «Sog. Volitionalität/Intentionalität», «Sog. allure extraordinaire» und «Sog. abtönende Verwendung» sind [187-190], erhalten Prädikate ohne Vorphase eine prospektiv ausgerichtete Vorphase [186]. ${ }^{33}$ Die allure extraordinaire und die «abtönende Verwendung», mit der Sokol Fälle wie Je vais vous demander und Je vous demanderai meint, ${ }^{34}$ werden nur sehr kurz [187-190] abgehandelt, wobei die allure extraordinaire zum Teil fehlerhaft dargestellt wird. ${ }^{35}$ Dabei ist der Begriff der «abtönenden Verwendung» für die Typen Je vais vous demander und Je vous demanderai nicht glücklich gewählt, da auch die allure extraordinaire auf Haltungen und Meinungen der Interaktanten verweist und damit ein abtönendes Verfahren ist (cf. Schrott 1997a, 262).

Von der semigrammatischen Periphrase unterschieden wird die prospektivinchoative Verwendung, die eine fortgeschrittenere Stufe der Grammatikalisierung darstelle, bei der dann auch Stativa und Progressiva, allerdings mit regelhafter inchoativ-transitioneller Reinterpretation, akzeptabel seien [190]. Als jüngster Funktionstyp wird dann die Tempusfunktion angeführt, bei der das $F P$ allein temporal-futurische Funktion habe und keinen Restriktionen bei den Prädikatklassen mehr unterliege, so daß auch Stativa und Progressiva ohne aspektuelle Umdeutung möglich seien [191]. In der Tempusfunktion habe das FP imperfektiven Wert [192] und entspreche dem imparfait (IMP). Zwar sei das FP noch nicht sehr weit in eine «explizit futurische und imperfektive Funktion» eingerückt, doch entwickle es sich zunehmend in diese Richtung [207]. ${ }^{36}$

Wie schon für das $F S$, so werden auch für das $F P$ bzw. für dessen Verwendungstypen die Interaktionen mit den verschiedenen Prädikatklassen be-

${ }^{33} \mathrm{Zu}$ diesen «Nutzwerten» ist anzumerken, daß Intentionalität bzw. Volitionalität sowohl vom $F S$ als auch vom FP in den entsprechenden grammatischen Personen (1. Person bzw. 2. Person) geleistet werden können, wobei die Futuropposition unterschiedliche Nuancierungen bedingt, cf. Schrott (1997a, 238-251).

34 Weiteres Material dazu bei Schrott (1997a, 318-323).

35 So entsteht der Wert der allure extraordinaire bei Negation durch eine externe Negation und nicht durch interne Negation, wie Sokol meint [187, 189]; cf. dazu Schrott (1997a, 270-284).

${ }^{36}$ Das in der Tempusfunktion für alle Prädikatklassen zugängliche FP könne damit anders als $F S$ und $P F$ «als einzige Form des Neufranzösischen eine unzweideutig futurische Funktion erfüllen» [195, cf. auch 203, 205]. Da in der Datenauswertung das FP im gesprochenen Teilkorpus häufiger auftritt als im geschriebenen Teilkorpus, schließt Sokol [195] unter der Prämisse, daß die zwei Register «zwei unterschiedliche Systemzustände» des Französischen anzeigen, auf eine zunehmende Verwendung des FP im heutigen Französisch. Diese Prämisse ist jedoch fraglich, zumal das geschriebene Teilkorpus im wesentlichen aus zwei zeitgenössischen Romanen und Pressetexten besteht. 
schrieben. Nach Sokol werden Stativa und Progressiva beim prospektiv-inchoativen Typ des FP transitionell umgedeutet, wogegen sie beim rein temporalen Verwendungstyp ohne Reinterpretation möglich seien, was belege, daß sich die Restriktion für Stativa in der Tempusfunktion auflöse [190]. Die Tatsache, daß Stativa wie savoir im FP sowohl mit stativischer Bedeutung ('Wissen haben') als auch mit transitioneller Umdeutung ('Wissen erwerben', 'erfahren') vorkommen können, ${ }^{37}$ wird damit durch die Existenz verschiedener Verwendungstypen mit unterschiedlichen Interaktionsmodi erklärt. Die entscheidende Frage ist nun, wie stichhaltig die Etablierung getrennter Verwendungstypen ist. So zeigen Beispielanalysen (Schrott 1997a, 113s.) und ergänzend durchgeführte analoge Sprecherbefragungen, daß transitionelle oder stativische Lesart immer auch entscheidend davon abhängen, ob der im Beispiel versprachlichte Sachverhalt im Kontext ein «Wissen-Haben» oder einen «Neuerwerb von Wissen» meint. Wie schon bei der Interaktion des FS mit Stativa, spricht auch im Fall des FP der Einfluß der Beispielkontexte gegen das Argument, daß sich die Lesarten exklusiv aus der Interaktion von Verbform und aktionaler Struktur ergeben und damit durch zwei getrennte, mit verschiedenen Interaktionsmustern verknüpfte Verwendungstypen erklärbar wären. ${ }^{38} \mathrm{Zu}$ überprüfen ist auch das Postulat, daß sich beim FP ein Verwendungstyp mit reiner Tempusfunktion und damit ohne prospektiv-inchoativen Aspekt herausbilde, in dem Stativa ohne Umdeutung möglich seien, wie das folgende Beispiel belegen soll [191]: ${ }^{39}$

(12) $[\ldots]$ viens manger, ça va être froid.

Das FP hat hier nach Sokol eine reine Tempusfunktion, in der «être froid» dann stativische Bedeutung habe. Diese Interpretation erfaßt jedoch die Funktion des FP im Kontext des Appells viens manger nicht, denn das FP als Ausdruck teilaktueller, im Werden begriffener Sachverhalte versprachlicht hier vielmehr, daß das Erkalten des Essens aktuell konditioniert ist und damit gleichsam bereits begonnen hat. ${ }^{40}$

Weitere Belege für eine rein temporale FP-Funktion glaubt Sokol [179] in drei analog funktionierenden Beispielen aus Schrott (1997a, 55; hier zit. nach Schrott) zu erkennen:

\footnotetext{
${ }^{37}$ Diese Koexistenz beider Bedeutungen belegen auch Beispielanalysen bei Schrott (1997a, 113s.).

${ }^{38}$ Die Korpusauswertung [192-194] ergibt nach Sokol, daß sich beim FP 11,17\% unbegrenzte Prädikate finden [195], wobei es in drei Fällen $(1,34 \%)$ zu einer inchoativtransitionellen Umdeutung komme. Für die Diskussion der von Sokol unterschiedenen Verwendungstypen wären diese Beispiele sämtlich von großem Wert, doch werden keine Belegstellen angeführt.

${ }^{39}$ Sokol führt zur Illustration des stativischen Werts insgesamt drei Beispiele aus dem Korpus ohne Quellenangabe an [191]. Die anderen zwei Beispiele erlauben aufgrund ihrer Kürze jedoch keine weitere beurteilende Untersuchung, cf. etwa das Beispiel «[...] tout le monde va avoir ce statut».

${ }^{40}$ Cf. auch die ähnlichen Beispiele (31) und (32) in Schrott (1997a, 47).
} 
(13) Ils n'arrivent pas à s'endormir. Va les embrasser, tu leur apprendras la bonne nouvelle. Ils (vont être si contents - seront si contents)!

(14) [...] Je le dirai à Madame $\mathrm{Ku}$, reprit vivement Alexandre. Elle (va être - sera) vexée, elle n'aimera pas ça, elle ne voudra plus te recevoir chez elle, il faut que tu viennes.

(15) Demain, j'emporterai le journal avec la vraie photo pour les montrer à M. Porteur. Il (ne va pas en revenir - n'en reviendra pas).

Die Tatsache, daß hier nach Sokol für das FP eigentlich restringierte Prädikate [179s.] auftreten, wird damit erklärt, daß das FP temporal-futurisch ohne aspektuellen Zusatzwert fungiere [180]. Doch die drei Beispiele führen diesen Beweis nicht. Zunächst illustrieren diese Beispiele den bereits erwähnten Fall, daß die im FP versprachlichten Sachverhalte von einer futurischen conditio abhängen (cf. Schrott 1997a, 56s.). Doch bleibt in allen drei Beispielen der Wert der aktuellen Konditioniertheit als meronymische Relation erhalten: das Überbringen der Neuigkeiten (13), (14) bzw. das Zeigen des Photos (15) erscheint fokussiert als Auslöser für emotionale Reaktionen. Diese Leistung des $F P$ wird auch kontrastiv zum in diesem Kontext ebenfalls sinnvollen FS deutlich, das in diesen Beispielen die zeitliche Sukzession betont. ${ }^{41}$

Die Möglichkeit, daß die hier dokumentierte Ausweitung des Prinzips der aktuellen Konditionierung beim FP in Zukunft zu einer Abschleifung des semantisch-pragmatischen Profils führen kann, stellt sicher eine denkbare Entwicklungslinie dar. Einen Beleg für einen solchen Verlust der meronymischen Semantik und des prospektiven Aspekts liefern die besprochenen Beispiele allerdings nicht, so daß das Postulat einer reinen Tempusfunktion des FP ungesichert erscheint.

\section{Synchronie, Diachronie und - Futurität: methodologische Reflexionen}

\subsection{Die Rolle der historischen Einzelsprache}

Die hier unternommene kritische Sichtung der Ausführungen zur Diachronie (3) sowie der Darlegung der synchronen Funktionsmuster (4) bei Sokol soll nun in methodologischer Perspektive resümiert werden. So ist deutlich geworden, daß die Abrisse der diachronen Entwicklung von FS und FP sich auf eine sehr schmale Datenbasis stützen und an entscheidenden Stellen mit übereinzelsprachlichen Tendenzen argumentieren. ${ }^{42}$ Bei Untersuchungen, die in hohem Maße das Besondere der Einzelsprachen dem Allgemeinen unter-

${ }^{41}$ Eine weitere temporal-futurische, nicht-prospektive Verwendung des $F P$ glaubt Sokol [192] auch in dem schon zitierten Beispiel (2) zu entdecken, in dem sie das FP als temporal-imperfektiv deutet, wobei die imperfektive Markierung dem Aspekt des IMP entspreche.

42 Studien wie Bybee/Perkins/Pagliuca (1994), die übereinzelsprachliche Tendenzen bei Tempus, Aspekt und Modalität herausarbeiten, sind sich im übrigen der Grenzen ihrer Untersuchung sehr wohl bewußt (1994: 36): «[...] we recognize better than anyone else possibly could how much we are limited by our source material». 
ordnen, ist jedoch die Gültigkeit der übereinzelsprachlichen Tendenzen zu hinterfragen, die sich in der Geschichte bestimmter sprachlicher Formen dokumentieren sollen. So wäre etwa zu präzisieren, um welchen Typ sprachlicher Universalien es sich bei solchen Tendenzen handelt (cf. Coseriu 1975, bes. 128-130). ${ }^{43}$ Die Frage nach der Gültigkeit ist besonders dringlich, wenn diese Tendenzen einzelsprachlich-diachrone Studien nicht nur ergänzen, sondern zum großen Teil ersetzen sollen. Denn um Sprachwandel angemessen zu beschreiben, sind neben allgemeinen Grundgegebenheiten des Sprachwandels als universales Phänomen auch empirische Daten historischer Einzelsprachen nötig (cf. etwa Lüdtke 1980, 182s.). Die sparsame Einbeziehung von spätlateinischen bzw. altfranzösischen Belegen ist auch deshalb befremdlich, weil die romanischen Sprachen durch ihre reich dokumentierte Sprachgeschichte einen Glücksfall für die historische Sprachwissenschaft und damit auch für die Grammatikalisierungstheorie darstellen. ${ }^{44}$

Im Unterschied zur Diachronie werden die synchronen Funktionsmuster der Futura von einer Korpusauswertung begleitet [cf. 81-89]. Da die Studie ein theoretisches Modell einzelsprachlich untermauern will, sollen die Hypothesen durch ein Korpus der französischen Gegenwartssprache «statistisch verifiziert bzw. rektifiziert» [12] werden. Untersucht werden drei Teilkorpora: ein literarisch-narratives Korpus, ein sprechsprachliches Korpus und ein pressesprachliches Korpus [81-83]. Das literarisch-narrative Korpus umfaßt zwei Romane unterschiedlicher stilistischer Prägung (Yann Queffélec: Les noces barbares; Georges Simenon: L'affaire Saint-Fiacre). Das sprechsprachliche Korpus enthält ausgewählte Transkriptionen aus den Korpora von Eschmann (1984) und Ludwig (1988), die den Rubriken «Informelle Gespräche», «Busfahrer-Funkkontakte» und «Mediendiskurse» zugeordnet werden [82s.] ${ }^{45}$ Die Teilkorpora sind also relativ klein, besonders schmal ist jedoch das pressesprachliche Teilkorpus (Le Monde 14./15. Juli 1996, p. 1-4; Les Échos. Le Quotidien de l'Économie, 12./13. Juli 1996, p. 1-5). ${ }^{46}$

Für die Auswertung werden die Daten nach aktionalen Klassen geordnet, wobei zwischen Verwendungen «ohne determinierenden Kontext» und Verwendungen, die «futurisch prädeterminiert» sind, unterschieden wird [84].

\footnotetext{
43 Sokol spricht an einer Stelle (ironisch?) von «allen ehernen Gesetzen der Grammatikalisierung» [127].

${ }^{44}$ Zur Problematik der Rekonstruktion diachroner Entwicklungen bei nicht-dokumentierten Sprachen meint etwa Haspelmath $(1998,58)$ : «In order to solve the remaining puzzles concerning the kind of change discussed here, one would have to look in greater detail at the individual languages whose earlier stages are attested and see whether the historical record contains any hints».

45 Nicht vertieft wird bei den Teilkorpora die Frage, inwieweit die geschrieben und gesprochen realisierten Texte der Nähesprache bzw. der Distanzsprache zuzuordnen sind. Sokol [81] verweist lediglich auf die Einschätzung «fiktiver Mündlichkeit» bei Koch/Oesterreicher (1990, 26).

${ }^{46}$ Dazu merkt Sokol [83] an, das Korpus sei «bewußt klein gehalten, um die registerübergreifende Statistik nicht zu stark zu beeinflussen oder gar zu verfälschen».
} 
Die Grundlage für die Bestimmung der aktionalen Klasse ist dabei nicht der Satz, wie er im Korpus vorkommt, sondern dessen Setzung in das als neutral eingestufte présent. $^{47}$ Obwohl Sokol auf einzelne problematische Fälle wie etwa idiomatische Wendungen im FS (Tu en verras d'autres) hinweist [86], wird das verzerrende Potential dieser Setzung in eine andere Verbform unterschätzt, denn die von ihr präsupponierte Unmarkiertheit des présent würde ebenfalls einen Wert darstellen, der den Satz beim Transfer verändern kann. Doch selbst wenn man von solchen Fehlerquellen absieht, können die durchgeführten Auswertungen ([81ss., 120s., 164ss., 192ss.]) die postulierte statistische Verifizierung der Hypothesen [12] nicht erbringen, da wichtige Grundprinzipien der schließenden Statistik nicht eingehalten werden. So enthält die betrachtete Stichprobe nicht die für statistische Verfahren notwendige Zahl an unabhängigen Beobachtungen. Die Datenerhebung liefert damit zwar ein anschaulich aufbereitetes Korpus, kann aber keine statistische Überprüfung der Hypothesen leisten. ${ }^{48}$

Da Sokol ihre Untersuchung in den einzelsprachlich orientierten Kapiteln als «Gegenstück und Ergänzung» [2] zu Schrott (1997a) konzipiert und engen Bezug auf die Beispieldiskussionen in meiner Studie nimmt, ist es folgerichtig und sinnvoll, daß ein nicht unerheblicher Teil der französischen Beispiele aus Schrott (1997a) stammt. Doch angesichts der Tatsache, daß Sokol über ein eigenes Korpus der französischen Gegenwartssprache verfügt, stellt sich die Frage, wieso die gesammelten Textbeispiele nicht stärker in die Ausführungen eingearbeitet wurden, sondern weitgehend auf die Abschnitte zur Datenerhebung beschränkt bleiben und bis auf sehr wenige Zitate nur in Form tabellarischer Auszählungen in Erscheinung treten.

Die von Sokol bei der Korpusvorstellung angeführten und diskutierten Beispielsätze aus dem Korpus [cf. 85-87] sind überwiegend sehr kurz gehalten, wobei die Quellen nicht genannt werden. ${ }^{49}$ Diese knappe Präsentation ist jedoch in einigen Fällen für das Verständnis der Interpretationen nicht unproblematisch. So deutet Sokol etwa das Beispiel La seconde messe est à sept heures wie folgt [87]: «Äußerungen wie die folgende waren ausschließlich über das außersprachliche Bild zu kategorisieren, für das sie stehen, in diesem Fall der (habituell?) wiederkehrende Zeitpunkt des Beginns der Früh-

\footnotetext{
47 Sokol [85]: «Die Grundlage für die Kategorisierung ist, wie gesagt, der entsprechende Satz im Präsens».

48 Cf. etwa Schwarze (1988, 13-15); Voß et al. (2000, 17-23, 47-50). Im allgemeinen benötigen statistische Verfahren mindestens 30 unabhängige Beobachtungen (cf. Voß et al. 2000, 293, 309ss.). So wären etwa 30 Romane verschiedener Autoren einer Epoche eine ausreichende Stichprobe. Das von Sundell (1991) untersuchte Korpus von 50 Romanen $(1991,13)$ stellt z. B. eine Stichprobe dar, die statistischen Anforderungen genügt.

${ }^{49}$ In anderen Fällen - etwa [155] (233) und [157] (243), (244) - wird bei den Korpusbeispielen zwar das Teilkorpus genannt, aus dem ein Beispiel stammt, doch unterbleibt ein genauerer Quellenverweis.
} 
messe. Damit handelt es sich aktional am ehesten um eine Transition mit Nachphasenimplikat». Diese abstrakte Deutung kann aber ohne weiteren Kontext bzw. ohne eine Quellenangabe, die eine Lektüre in einem weiteren Kontext ermöglichen würde, kaum eingeschätzt und bewertet werden. ${ }^{50}$ In solchen Fällen erschwert die knappe Beispielpräsentation dem Leser die verstehende Beurteilung der Interpretationen.

Zur Erläuterung der Beispiele gibt Sokol in vielen Fällen Paraphrasen und Übersetzungen an, die aber nicht immer überzeugen. So trifft etwa die Deutung, Vous remarquerez que je suis patiente (Beispiel nach Schrott 1997a, 255) sei als rezente Vergangenheit zu interpretieren («Sie haben sicher bemerkt, daß ich geduldig bin») [154], nicht zu, denn das FS funktioniert hier (analog zu Vous m'excuserez) virtuell-futurisch und versprachlicht einen dem FS vorbehaltenen Verwendungstyp zum Ausdruck von Handlungen, die Konsens und freien Willen des Adressaten erfordern (cf. dazu Schrott 1997a, 253256). Und auch bei den Beispielen Jeanne jouera du piano [152] und Il saura la réponse [155] werden die von Sokol angegebenen modalen Paraphrasen «Jeanne ist wohl Klavierspielerin» bzw. «Er wird die Antwort vermutlich wissen» von befragten Muttersprachlern überwiegend nicht akzeptiert und erscheinen so zumindest fragwürdig. ${ }^{51}$

Kritisch anzumerken ist auch, daß Sokol in ihren Ausführungen zur Interaktion von Aspekt und aktionalen Klassen relativ häufig auf deutsche Beispiele rekurriert, und zwar nicht nur im allgemeinen theoretischen Teil, sondern auch in den Kapiteln, die den französischen Tempusformen gewidmet sind. So wird etwa im Kapitel zum $P F$ [cf. 91-113] die Diskussion um die Aspektmarkierung und das Verhalten aktionaler Klassen zu einem großen Teil anhand deutscher Beispiele geführt (cf. auch die Kritik von Werner 2001, 351). Diese bereits erwähnte Vermischung einzelsprachlicher Systeme ist wegen der ungesicherten Analogie zwischen den verschiedenen einzelsprachlichen Tempus-Aspekt-Systemen jedoch ein methodologisch prekäres Verfahren. ${ }^{52}$

\subsection{Konditioniertheit - Aspekt - Futurität}

Nach Sokol können die in Schrott (1997a) gemäß dem «Prinzip der Konditionierung» formulierten Basiswerte der Futura als «Koppelung und Zusammen-

\footnotetext{
${ }^{50}$ Allenfalls Leser, die wissen, daß im Korpustext L'affaire Saint Fiacre das Opfer während einer Messe zu Tode kommt, haben eine Chance, das Zitat mit Ausdauer und Scharfsinn (Simenon!) aufzuspüren. In der Presses-Pocket Ausgabe (Paris, 1976) findet sich das Zitat auf p. 18.

${ }^{51}$ In einigen Fällen sind die gegebenen Beispiele auch fehlerhaft: [108]: Il trouvais [sic] ses clés lorsque le téléphone sonna; Il tirais [sic] lorsque le téléphone sonna; [123]: Personne ne te crô̂t [sic]; [141]: Marcher, cueuillir [sic] les fleurs [...].

${ }^{52}$ So lehnt Sokol etwa das Konzept eines Plans beim französischen $P F$ unter Verweis auf das deutsche praesens pro futuro in einem argumentativen «Sprung» zwischen den historischen Einzelsprachen ab [42]: «Leider gilt dies für das Deutsche nicht, und das Argument ist von dieser Seite her betrachtet bereits anfechtbar».
} 
spiel grundlegender Merkmale» von Aspekt und aktionalen Strukturen gedeutet werden [208]. In ihren Augen ist die Differenzierung von aktueller und virtueller Konditionierung damit ein Epiphänomen der Interaktion von Aspekt und aktionalen Klassen. Setzt man wie Sokol diese Interaktion als primäre Kraft an, dann müssen sich Fälle finden, in denen das Modell der virtuellen bzw. aktuellen Konditioniertheit die Futurverwendung nicht erklären kann, wohl aber das Modell von Aspekt und aktionalen Strukturen. Im Fall des FS ist nach Sokol die virtuelle Lesart «in vielen Fällen» möglich [146] - die Formulierung legt nahe, daß die virtuelle Lesart nicht in allen Fällen möglich sei, wobei allerdings etwaige Gegenbeispiele nicht genannt werden. Beim FP dagegen führt Sokol Fälle an, deren «Diskurswert» nicht über die aktuelle Konditionierung erklärt werden könne [205]. Die Diskussion der Beispiele für eine solche temporal-futurische, aspektuell unmarkierte Verwendung zeigte jedoch, daß dieser Verwendungstyp nicht ausreichend belegt werden kann.

Um die Erklärungskraft beider Modelle auszuloten, sollen die Funktionsmuster von FS und FP vergleichend-kontrastiv durch Kommutationen in Beispieltexten herausgearbeitet werden. Diese Verzahnung der Futuropposition gerät bei Sokol gar nicht erst ins Blickfeld der Untersuchung, weil FS und FP (und ebenso das $P F$ ) als isolierte Paradigmen untersucht werden, deren Profile nicht systematisch kontrastiert werden. Greift man nun Beispiele heraus, in denen nur das FS bzw. nur das FP akzeptabel sind, dann zeigt sich, daß das Prinzip der Konditioniertheit höhere Erklärungskraft hat als das Aspektmodell: 53

(16) «Mais moi...», cria Paul. Il ne put achever sa phrase, car elle venait de serrer sur sa nuque le nœud de sa serviette, et elle ajouta: «Finis de manger ta soupe, tu (parleras - *vas parler) après».

Die exklusive Akzeptanz des FS kann hier über die Interaktion von Aspekt und aktionalen Strukturen nicht erklärt werden. Die aktionale Struktur von «parler» (Sokol würde vom Satz Tu parles im présent als Reinform der aktionalen Struktur ausgehen) entspricht in Sokols Einteilung einem Progressiv. Nach Sokol löst das FS bei Progressiva primär eine iterative Lesart aus, doch sind auch futurische Werte möglich [151, 152, 207] - letztere Lesart würde vorliegen, denn eine iterative Deutung scheidet im gegebenen Kontext aus. Das von befragten Sprechern abgelehnte $F P$ müßte gemäß den postulierten Interaktionsmustern von Aspekt und aktionalen Strukturen aber ebenfalls möglich sein. Denn als rein temporale Futurform ist das FP mit allen Situationstypen kompatibel [191], und auch als prospektiv-inchoativer Funktionstyp - wenn man Sokols Einteilung zugrundelegt - wäre das FP möglich, da Progressiva nach Sokol bei dieser Funktion mit transitioneller Reinterpretation [190] akzeptabel sind. Aus Aspekt und aktionalen Strukturen kann also

53 Beispiel aus: Pagnol, Marcel, Le temps des secrets. Souvenirs d'enfance, Paris, Éditions de Fallois, [1960] 1988, 186; zit. nach Schrott (1997a, 33). 
nicht die Restriktion hergeleitet werden, die sich in der Sprecherbefragung deutlich zeigt. Dagegen kann das Prinzip der Konditionierung den Ausschluß des FP erklären. Aus der Situation geht nämlich hervor, daß Paul sich erst wieder zu Wort melden darf, wenn er seine Suppe gegessen hat. Da diese Bedingung in der Referenzsituation, die auch die Sprechsituation ist, noch nicht erfüllt ist, hängt die futurische Handlung von einer virtuellen und noch unerfüllten conditio ab - nur das FS kann diese Virtualität und damit die in diesem Kontext sinnvolle Nuance ausdrücken. $\mathrm{Zu}$ fragen ist hier auch, wie über die Interaktion von Aspekt und Aktionsart Fälle erklärt werden sollen, bei denen FS und FP möglich sind, allerdings mit ganz verschiedenen Nuancen (Schrott 1997a, 33s.), wie das im anfangs zitierten Beispiel (2) der Fall war. Das Prinzip der Konditionierung kann hier nicht nur erklären, wieso sowohl FP als auch FS möglich sind, sondern auch wieso die Kommutation durch das FS eine veränderte Nuance ergibt.

Wie kann das Prinzip der Konditioniertheit nun mit dem Konzept aspektueller Markierungen verbunden werden? Grundsätzlich wird die aktuelle bzw. virtuelle Konditioniertheit als ein Grundwert verstanden, der sich in verschiedenen sprachlichen Subsystemen - etwa bei den Aktanten (Schrott 1997a, 85-95) - manifestiert und mit verschiedenen Modellen beschreibbar ist. So werden in Schrott $(1997 \mathrm{a}, 141)$ die aspektuellen Markierungen von FS und FP als Manifestationen der Konditionierung aufgefaßt: das FP hat durch die aktuelle conditio einen prospektiven Aspekt, der einen Sachverhalt als beginnendes, sich entwickelndes Geschehen auffaßt, während das virtuell konditionierte FS keine solche interne Struktur beinhaltet. Ausgehend von Comries übereinzelsprachlich konzipierten Definitionen von perfektivem und imperfektivem Aspekt wird das prospektive FP, das die innere Sachverhaltsstruktur fokussiert, in Schrott (1997a, 110) als imperfektive Form gedeutet, während das FS, das keine solche Fokussierung leistet, als perfektiv charakterisiert wird.$^{54}$ Erstaunlich ist nun, daß trotz der Verschiedenheit der Modelle den Futura bei Sokol und in Schrott (1997a) zum Teil kongruente Aspektmarkierungen zugewiesen werden. Wie Schrott (1997a, 110), so stuft auch Sokol das FS als perfektive Form ein, mit dem Unterschied, daß sie die Form nicht als Futurform, sondern als Aspekt auffaßt, der dann bei bestimmten aktionalen Klassen futurische Funktion habe [165]. ${ }^{55}$ Weniger festgelegt erscheint dagegen bei Sokol die Aspektmarkierung des FP. So versprachliche die aller-

${ }^{54}$ Zur Aspektmarkierung cf. Comrie (1976, 4-5 2-23, 24s., 64); cf. Schrott (1997a, 101-117, bes. 107-109 und 110); cf. auch Togeby (1982, 317), der das FS ebenfalls als perfektiv einstuft.

${ }^{55}$ Ein perfektiv markiertes Futur stellt übrigens nach Bybee/Perkins/Pagliuca (1994) einen Sonderfall dar, denn die Datenauswertung zeige, daß «perfectives» typischerweise vergangene Sachverhalte beschreiben (1994, 83, cf. auch 175) und perfektive Aspekte selten «future uses» aufweisen (1994, 278): «Perfectives with future uses are even less common than imperfectives, as we would expect, given the widespread restriction of perfectives to the past». 
Periphrase in lexikalischer Vollbedeutung und in semigrammatischer Bedeutung ein Ereignis, das «implizit perfektiv von außen kommuniziert» sei [204]. Die inchoative Funktion werde «in erster Linie als perfektiv aspektuell und nur implizit futurisch dekodiert» [205], wogegen der Tempusfunktion ein futurisch-imperfektiver Wert [192, 205] zugeschrieben wird. Damit haben die synchronen Verwendungstypen des FP nach Sokol perfektive und imperfektive Lesarten, wobei das FP in seiner «statistisch häufigsten Variante» grenzbezogen perfektiv sei [209].

Die zentrale Frage ist nun, auf welcher Basis solche aspektuellen Charakterisierungen der französischen Futura vorgenommen werden. Zunächst repräsentieren die (in der Tradition Comries stehenden) Definitionen des imperfektiven und des perfektiven Aspekts als Innenperspektive bzw. Außenperspektive ein übereinzelsprachliches Modell, das zeitliche Konturen in einer räumlichen Metapher darstellt. ${ }^{56}$ Betrachtet man nun einzelsprachliche Studien zum Aspekt im Französischen, dann wird diese Aspektopposition meist anhand der Vergangenheitstempora passé simple (PS) und imparfait (IMP) untersucht. ${ }^{57} P S$ und IMP gelten dann als Manifestationen von Perfektivität und Imperfektivität im Französischen, zu denen FS und FP in Analogie gesetzt werden. Aufgrund dieser Überschneidung sollte bei aspektuellen Charakterisierungen der Futura immer klargestellt werden, ob ein übereinzelsprachliches Konzept oder eine einzelsprachliche Analogie zugrunde gelegt wird. Beide Optionen können Unschärfen mit sich bringen. So kann die räumliche Metapher des übereinzelsprachlichen Konzepts von Außensicht und Innensicht das Funktionieren von $P S$ und IMP nur zum Teil erklären. Denn während das IMP Sachverhalte erfaßt, die ohne Begrenzung gesehen werden («Innenperspektive»), versprachlicht das PS Sachverhalte, die in der Vergangenheit begrenzt sind («Außenperspektive») und die diesseits und/oder jenseits dieser Grenze in der Vergangenheit noch nicht oder nicht mehr bestanden (cf. Morgenstern 1986, 80s.). Diese (Nicht-)Begrenzung bewirkt, daß Sachverhalte im $P S$ unter dem Aspekt der Veränderbarkeit der Vergangenheit gesehen werden, wogegen das IMP Sachverhalte versprachlicht, die nicht veränderbar sind bzw. nicht unter dem Aspekt der Veränderbarkeit gesehen werden. Bei Iterationen drückt das IMP unbegrenzt oft iterierende Sachverhalte aus, wogegen das PS begrenzt iterierende Sachverhalte wiedergibt. Die Metapher der Perspektive kann die Merkmale der Begrenzung (Außensicht) bzw. der NichtBegrenzung (Innensicht) zwar veranschaulichen, doch werden ebenfalls wichtige Charakteristika wie die (begrenzte oder unbegrenzte) Iteration und die Qualität der Veränderbarkeit nicht adäquat wiedergegeben. ${ }^{58}$ Ein weiteres Ar-

56 Comrie (1976, 4, 17-19, 21ss., 24s.); Schrott (1997a, 101-103); cf. auch die schon erwähnte Aspekt-Definition bei Sokol [54s.].

57 Cf. zum Aspekt bei den französischen Vergangenheitstempora Mitko (2000, 514, 17-51).

${ }^{58}$ Diese Ungenauigkeiten sind $\mathrm{m}$. E. auch der Tatsache geschuldet, daß das spatiale Modell eines perspektivierenden Aspekts nicht impliziert, daß der Aspekt selbst 
gument für einen vorsichtigen Transfer von übereinzelsprachlichen AspektKonzepten auf die historischen Einzelsprachen liefert die sprachvergleichende Studie Iva Novakovas (2001) zu den Futura im Französischen und Bulgarischen, die anhand zahlreicher Übersetzungsvergleiche (2001, 205-298) überzeugend belegt, daß weder das FS noch das FP in ihrem aspektuellen Potential auf den perfektiven oder imperfektiven Aspekt festgelegt sind (bes. 2001, 212s., 234s., 248, 264s.), sondern daß bei FS und FP vielmehr die Opposition von «rupture» und «continuité» distinktiv für die Tempuswahl ist.

Können solche Unschärfen vermieden werden, wenn die Aspektmarkierungen der Futura in einzelsprachlicher Analogie zu PS und IMP beschrieben werden? So setzt etwa Sokol [192] die imperfektive Markierung des FP mit dem imperfektiven Aspekt des IMP gleich. ${ }^{59}$ Sinnvoll ist ein Transfer der für $P S$ und IMP formulierten Aspektoppositionen auf die Futura natürlich nur dann, wenn es eine Symmetrie zwischen den Vergangenheitstempora und den Futura gibt und sich zwischen $P S$ und FS bzw. zwischen IMP und FP kongruente Aspektmarkierungen etablieren lassen. Dies ist aber nur zum Teil der Fall. So ist IMP und FP zwar gemeinsam, daß sie keine Zieleinlösung versprachlichen, sie unterscheiden sich aber hinsichtlich zweier Charakteristika. So strebt das FP eine Zieleinlösung an (prospektiver Aspekt), was beim IMP nicht der Fall sein muß, und das $F P$ hat eine Anfangsterminierung, während es für das IMP gerade typisch ist, daß es eben keine Anfangsterminierung (und auch keine Endterminierung) hat. FS und PS haben zwar gemeinsam, daß sie Sachverhalte ohne Fokussierung interner Strukturen versprachlichen und als Formen mit perfektiver Außenperspektive aufgefaßt werden können, doch müßte die Gleichwertigkeit der Aspektmarkiertheit noch einzelsprachlich untermauert werden. Zudem hat das Prinzip der aktuellen bzw. virtuellen Konditionierung im Bereich der Vergangenheitstempora kein Äquivalent, sondern ist spezifisch für die Tempora der Zukunft, was den Schluß nahelegt, daß im Französischen für die Futuropposition andere distinktive Kriterien gelten als für die Opposition der Vergangenheitstempora.

Die folgenden Beispiele kontrastieren die Aspektmarkierungen von FS und $F P$ sowie von $P S$ und IMP und liefern deutliche Indizien dafür, daß Futura und Vergangenheitstempora nicht mit den gleichen Aspekt-Etiketten versehen werden sollten. Ersetzt man im bereits zitierten Beispiel (2), das Sokol [192, zit. nach Schrott 1997a] als Beleg für den imperfektiven Wert des FP versteht, das FP durch das IMP, dann wird die Nichtäquivalenz der Formen in der Beispielvariante deutlich:

eine räumliche Kategorie ist. Denn die metaphorische Spatialisierung liegt zunächst einmal im sprachwissenschaftlichen Modell und darf nicht in einem transitus ab intellectu ad rem auf das Objekt der Untersuchung übertragen werden.

${ }^{59}$ Sokol bezieht mehrfach die Vergangenheitstempora $P S$ und IMP vergleichend ein (n. 18 [102], n. 20 [103]; n. 22 und 23 [108]); auch Schrott (1997a, 171 n. 333) zieht eine Parallele zwischen $P F$ und $I M P$. 
(17) Nous passions des vacances magnifiques! Nous mangions de belles brochettes de grives, de darnagas et de perdrix!

Das IMP versprachlicht hier einen Sachverhalt, der schon und noch existierte, sei es als bereits gegebener Zustand, sei es als unbegrenzte Iteration ('Immer, wenn wir in die Ferien fuhren...'). Im Originaltext (2) dagegen kann das FP keine unbegrenzte Iteration versprachlichen, und auch die imperfektive Markierung eines schon und noch gegebenen Sachverhalts liegt beim FP nicht vor, da das FP aktuell konditionierte und damit als Vorstufe anfangsterminierte Sachverhalte ausdrückt, wogegen beim IMP Anfang und Ende ja gerade ausgeblendet werden.

Die Inkongruenz der einzelsprachlichen Aspekt-Analogie zeigt sich noch deutlicher, wenn man FS- und FP-Verwendungen in der Funktion futur historique in erzählend-berichtenden Passagen mit den Vergangenheitstempora kommutiert. Im Falle gleichwertiger Aspektmarkierungen müßte das als prospektive Form imperfektiv gedeutete $F P$ stets dem IMP entsprechen, das als perfektiv etikettierte $F S$ dagegen wäre durch das $P S$ wiederzugeben. Dazu einige Beispiele aus einem Geschichtsbuch, ${ }^{60}$ bei dem die futur historiqueVerwendungen (abgesichert durch Sprecherbefragungen) durch IMP und PS kommutiert wurden. Zunächst ein Beispiel zum FP, das aspektuell dem IMP entsprechen müßte, im folgenden aber nur durch das $P S$ ersetzbar ist:

(18) La vigueur du sentiment national vient de se révéler. Mais Henri III et ses successeurs vont, pendant plus de cent ans, disputer la couronne de France aux Valois, rois selon le cœur des Français (Clozier/Depain/Guyomard 1954, 102).

(19) Mais Henri III et ses successeurs (*disputaient - disputèrent) pendant plus de cent ans la couronne de France aux Valois, [...].

Wie die nächsten Beispiele zeigen, ist auch das FS aspektuell nicht dem PS äquivalent, denn es finden sich sowohl Beispiele, in denen es allein durch das $P S$ ersetzt werden kann, als auch Textbeispiele, in denen nur durch das IMP kommutiert werden kann:

(20) Préparée par Henri IV et Richelieu, la monarchie absolue est organisée par Louis XIV. Ce régime durera (dura - *durait) plus d'un siècle [...] (Clozier/Depain/Guyomard 1954, 168).

(21) Aussi, quand le vainqueur revenait dans sa ville, on l'y recevait en triomphe, vêtu de pourpre, sur un char traîné par quatre chevaux et, toute sa vie, il restera (restait - *resta) un personnage entouré du respect de la cité, [...]. Il aura (avait - *eut) même droit à l'érection d'une statue à Olympie (Clozier/Depain/Guyomard 1954, 15).

Während in (20) nur das PS möglich ist, erfordert der Textausschnitt (21) das $I M P$ : beschrieben wird hier nämlich der Brauch, bei den Olympischen Spielen siegreiche Athleten zu ehren, so daß eine unbegrenzte Iteration vorliegt.

${ }^{60}$ Clozier, René/Depain, Henri/Guyomard, Yves, La France dans l'histoire de la civilisation, Paris, Larousse, 1954. 
Diese Transpositionen der Futurverwendungen in die Vergangenheit belegen, daß keine aspektuellen Äquivalenzen zwischen FS und PS einerseits sowie FP und IMP andererseits bestehen, weshalb Parallelen zwischen den Futura und den Vergangenheitstempora nur mit großer Umsicht gezogen werden sollten. Doch nicht nur einzelsprachliche Analogien sind problematisch, auch der Transfer der übereinzelsprachlichen Aspektopposition von Außen- und Innenperspektive auf die Futura, wie er sich bei Sokol und in Schrott (1997a) findet, kann Unschärfen mit sich bringen.

\section{Konklusion}

In ihrer Studie setzt sich Monika Sokol das Ziel, Theoriebildung und einzelsprachliche Analyse in einem Brückenschlag zu verbinden und ein übereinzelsprachliches Modell am Beispiel des Französischen zu exemplifizieren [3s.]. Die ihren Analysen zugrundeliegende Kombination von übereinzelsprachlichen Regeln, diachronen Ausgriffen und synchroner Datenerhebung öffnet den Blick für anregende Hypothesen zu Aspekt und aktionalen Strukturen, doch kann, wie die kritische Sichtung zeigte, in den meisten Fällen die postulierte einzelsprachliche Überprüfung nicht geleistet werden. So stützen die Abhandlungen zur Diachronie sich in großen Teilen auf übereinzelsprachliche Tendenzen, nutzen jedoch das Reservoir der spätlateinischen bzw. altfranzösischen Quellen und Belege kaum. Eine solche bei nicht dokumentierten Sprachen notgedrungen betriebene konjizierte Geschichtsschreibung («conjectural history») kann aber in der romanistischen Sprachgeschichtsschreibung mit ihrer privilegierten Datenlage nur geringe Berechtigung einfordern. Anders gestaltet sich das Bild bei den synchronen Funktionsmustern, die durch französische Beispiele illustriert und durch Korpusauswertungen dokumentiert werden. Doch finden sich auch hier Faktoren, die das Gewicht des einzelsprachlichen Materials mindern. So leistet das relativ kleine Korpus eine Veranschaulichung, nicht aber die von Sokol in Aussicht gestellte statistische Verifizierung ihrer Hypothesen.

Ein nicht nur methodisches Problem der Studie stellt auch der Transfer zwischen den Einzelsprachen dar, etwa wenn Argumentationen gleichermaßen auf das Deutsche und das Französische rekurrieren. Denn eine Darstellung des Funktionsspektrums einer Form, die Belege aus verschiedenen Sprachen mischt, fokussiert primär allgemeine Entwicklungslinien, nicht aber das besondere Profil einer bestimmten einzelsprachlichen Form. ${ }^{61}$ Das anvisierte Ziel, ein übereinzelsprachliches Modell am Beispiel des Französischen in Diachronie und Synchronie zu exemplifizieren, würde jedoch ein Gleichgewicht zwischen dem Besonderen und dem Allgemeinen erfordern. Denn auch Sprachwissenschaftler, die wie Meisel/Schwarze $(2002,435)$ in ihrem pro-

61 Cf. auch die Kritik von Werner (2001, 351), das Französische erscheine «im Dienste einer allgemeinen sprachwissenschaftlichen Fragestellung instrumentalisiert». 
grammatischen Aufsatz über «Das Besondere und das Allgemeine» fordern, daß Einzelsprachen als Manifestationen der universellen faculté du langage zu analysieren seien, betonen, daß zugleich auch immer die (möglichen) Universalien an einzelsprachlichen Befunden gemessen werden müssen. Auch in der Sichtweise einer die Strömungen der Allgemeinen Sprachwissenschaft aufnehmenden Romanistik erscheint es also unabdingbar, daß das in der Tradition der romanistischen (und damit auch der historischen) Sprachwissenschaft erworbene Wissen systematisch eingespeist wird (Meisel/Schwarze 2002, 436-437). ${ }^{62}$

Abschließend kehre ich zu den beiden eingangs erwähnten Kernfragen zurück, denen Sokol erstmals systematisch nachgeht. Die erste Frage [6] gilt den Aspektoppositionen im futurischen Bereich. Die Reflexionen zur Etikettierung von $F S$ und $F P$ als perfektiv bzw. imperfektiv haben gezeigt, daß der Erkenntnisgewinn dieser mit mehreren Unschärfen verknüpften Zuordnung in Zweifel gezogen werden kann. Das Prinzip der Konditionierung kann zwar als aspektuelle Opposition (cf. Schrott 1997a, 110, 41) aufgefaßt werden, doch die von der Beschreibung der französischen Vergangenheitstempora geprägte Opposition von perfektivem und imperfektivem Aspekt suggeriert Äquivalenzen zwischen Futura und Vergangenheitstempora, die nicht vorhanden sind. Die Futura sollten daher nicht in das Prokrustesbett der anhand der Vergangenheitstempora entwickelten Aspekt-Definitionen gezwängt werden. Die Beschreibung der Futura als perfektiv bzw. imperfektiv markierte Formen leistet eine Charakterisierung der beiden Formen, sie ist aber kein distinktives Merkmal der Futuropposition. Begreift man perfektiven und imperfektiven Aspekt als übereinzelsprachlich, dann kann die Zuordnung für einen Sprachvergleich mehrerer Einzelsprachen sinnvoll sein. Dabei ist allerdings zu beachten, daß eine übereinzelsprachliche Definition keine Größe ist, die sich tel quel in den Einzelsprachen findet, sondern ein tertium comparationis, vor dessen Folie Unterschiede zwischen den Einzelsprachen plastisch werden.

Wie gestaltet sich im Spiegel des Besprechungsaufsatzes die Antwort auf die zweite Kernfrage Sokols nach den Affinitäten bzw. Restriktionen zwischen futurisch fungierenden Formen und bestimmten aktionalen Strukturen? Die Diskussion einer wichtigen Prämisse Sokols - der kognitive Status aktionaler Strukturen - führte vor Augen, daß das Leistungsvermögen kognitiver und pragmatischer Ansätze genau zu bedenken ist, und rührte an die zuletzt im Bereich der historischen Semantik intensiv diskutierte Frage, wie Sprachwissenschaftler Zugang zu kognitiven Konzepten finden können, die Sprechen und Verstehen sowie den Sprachwandel beeinflussen (cf. die programmati-

62 Diese Forderung nach einer Ausrichtung am einzelsprachlichen Material würde sich natürlich in der Perspektive einer auf die Romanistik zentrierten Sprachwissenschaft, wie sie etwa Kramer $(2004,68,72$ s.) in seinem (auf Meisel/Schwarze 2002 replizierenden) «Plädoyer für die Bevorzugung des Besonderen vor dem Allgemeinen» fordert, noch ungleich schärfer stellen. 
schen Ausführungen von Lebsanft/Gleßgen [2004, 12-18, bes. 14, 17s.] zur kognitiven und pragmatischen Semantik). Stützen sich Sprachwissenschaftler in zentralen Aspekten auf kognitive Parameter, dann müssen Ergebnisse und Methoden der Kognitionswissenschaften auch interdisziplinär und transdisziplinär integriert werden.

Für die diachrone Entwicklung der Futurformen, die Sokol als Geschichte der Interaktion von Aspekt und aktionalen Strukturen zu erklären sucht, muß die Frage nach Affinitäten und Restriktionen in früheren Stadien der Sprachgeschichte offen bleiben - das in der Studie angeführte Material reicht zur Klärung dieser Frage in der Diachronie schlichtweg nicht aus. Die in der Studie formulierten Hypothesen können daher nicht in den Stand sprachgeschichtlicher Fakten erhoben werden, denn «[o]hne exakte Übersicht über die sprachliche Wirklichkeit bleiben Hypothesen hypothetisch» (Müller 1969, 416).

Im Fall von FS und FP im Französischen der Gegenwart hat die Besprechung der Beispiele - vor allem derjenigen Beispiele, die Restriktionen belegen sollen - gezeigt, daß FS und FP für alle aktionalen Strukturen offen sind. ${ }^{63}$ Was die für $F S$ und $F P$ von Sokol postulierten systematischen Interaktionen zwischen Aspekt und aktionalen Klassen angeht, so legten die Beispieldiskussionen doch zahlreiche Abweichungen von den angenommenen Interaktionsmustern offen, weshalb die Existenz fester Automatismen zwischen Aspekt und aktionaler Struktur in Zweifel gezogen werden kann. Die beobachtbaren Wechselwirkungen und Umdeutungen im Zusammenspiel von Aspekt und aktionalen Klassen sind keine (allein) einzelsprachlich fixierten Muster, sondern hängen immer auch von den Verwendungskontexten ab: sie gehören damit nicht der Ebene der Einzelsprache an, sondern der Ebene der Texte und ihrer Kontexte. Damit sind diese Interaktionen jedoch ein Phänomen, das in den Bereich der Pragmalinguistik als der Beschäftigung mit der Rede in ihren Kontexten hineinstrahlt. Die sprachwissenschaftliche Pragmatik kann so auch für Studien zu Aspekt und aktionaler Semantik ein Leitstern sein.

\section{Literatur}

Aschenberg, Heidi, Kontexte in Texten. Umfeldtheorie und literarischer Situationsaufbau, Tübingen, Niemeyer, 1999.

Benveniste, Émile, Mutations of Linguistic Categories, in: Lehmann, W. P./Malkiel, Yakov (edd.), Directions for Historical Linguistics. A Symposium, Austin/London, University of Texas Press, 1968, 83-94.

Blank, Andreas, Prinzipien des lexikalischen Bedeutungswandels am Beispiel der romanischen Sprachen, Tübingen, Niemeyer, 1997.

Bonnard, Henri/Régnier, Claude, Petite grammaire de l'ancien français, Paris, Éditions Magnard, 1991.

63 Dies bestätigt auch die Studie Novakovas (2001, 205-298, bes. 210s., 222, 234s., 245, 264s.): Kontrastiv zum Bulgarischen zeigt sich, daß im Französischen die Verben in sämtlichen tiroirs verbaux auftreten können und auch bei $F S$ und $F P$ keinen allein der aktionalen Semantik geschuldeten Restriktionen unterliegen. 
Brüser, Martina, Die Verbalperiphrase aller + Infinitiv des Französischen und ihre Relation zum futur simple. Geschichte der Verbalperiphrase und synchrone Untersuchung unter besonderer Berücksichtigung des futur historique, Erlangen, 1989 [unveröffentlichte Magisterarbeit].

Bybee, Joan/Perkins, Revere/Pagliuca, William, The Evolution of Grammar. Tense, aspect and modality in the languages of the world, Chicago/London, The University of Chicago Press, 1994.

Comrie, Bernard, Aspect. An Introduction to the Study of Verbal Aspect and Related Problems, Cambridge et al., Cambridge University Press, 1976.

Coseriu, Eugenio, Determinación y entorno. Dos problemas de una lingüística del hablar, RJ 7 (1955-1956), 29-54.

Coseriu, Eugenio, Die sprachlichen (und die anderen) Universalien, in: SchliebenLange, Brigitte (ed.), Sprachtheorie, Hamburg, Hoffman und Campe, 1975, 127-161.

Coseriu, Eugenio, Sprachkompetenz. Grundzüge der Theorie des Sprechens, bearbeitet und herausgegeben von Heinrich Weber, Tübingen, Francke, 1988.

Detges, Ulrich, Wie entsteht Grammatik? Kognitive und pragmatische Determinanten der Grammatikalisierung von Tempusmarkern, in: Lang, Jürgen/NeumannHolzschuh, Ingrid (edd.), Reanalyse und Grammatikalisierung in den romanischen Sprachen, Tübingen, Niemeyer, 1999, 31-52 [1997 Paper zum Deutschen Romanistentag in Jena].

Eschmann, Jürgen, Texte aus dem «français parlé», Tübingen, Narr, 1984.

Fleischman, Suzanne, The Future in Thought and Language. Diachronic Evidence from Romance, Cambridge et al., Cambridge University Press, 1982.

Gougenheim, Georges, Étude sur les périphrases verbales de la langue française, Paris, Les Belles Lettres, 1929.

Haspelmath, Martin, The semantic development of old presents, new futures and subjunctives without grammaticalization, Diachronica 15 (1998), 29-62.

Koch, Peter/Oesterreicher, Wulf, Gesprochene Sprache in der Romania: Französisch, Italienisch, Spanisch, Tübingen, Niemeyer, 1990.

Kramer, Johannes, Linguistische Romanistik heute. Plädoyer für die Bevorzugung des Besonderen vor dem Allgemeinen, RF 116 (2004), 66-73.

Lausberg, Heinrich, Romanische Sprachwissenschaft, vol. 3: Formenlehre, Berlin/ New York, de Gruyter, ${ }^{2} 1972$.

Lebsanft, Franz/Gleßgen, Martin-Dietrich, Historische Semantik in den romanischen Sprachen. Kognition, Pragmatik, Geschichte, in: iid. (edd.), Historische Semantik in den romanischen Sprachen, Tübingen, Niemeyer, 2004, 1-28.

Lehmann, Christian, Thoughts on Grammaticalization, München/Newcastle, Lincom Europa, 1995.

Ludwig, Ralph, Korpus: Texte des gesprochenen Französisch. Materialien I, Tübingen, Narr, 1988.

Lüdtke, Helmut, Auf dem Wege zu einer Theorie des Sprachwandels, in: id. (ed.), Kommunikationstheoretische Grundlagen des Sprachwandels, Berlin/New York, de Gruyter, 1980, 182-266.

Meibauer, Jörg, Rhetorische Fragen, Tübingen, Niemeyer, 1986.

Meisel, Jürgen M./Schwarze, Christoph, Romanistische Linguistik heute. Das Besondere und das Allgemeine, RF 114 (2002), 423-444.

Mitko, Julia, Aspekt im Französischen. Eine semantisch-funktionelle Studie, Tübingen, Narr, 2000.

Morgenstern, Claus, Rez. zu Togeby, Knud, Grammaire française, vol. 2, 1982, ZfSL 96 (1986), 75-86.

Müller, Bodo, Das lateinische Futurum und die romanischen Ausdrucksweisen für das futurische Geschehen, RF 76 (1964), 44-97.

Müller, Bodo, Futur und Virtualität, ZrP 85 (1969), 416-427. 
Müller, Bodo, Die Probleme des romanischen Futurs.(Auch eine Erwiderung), ZrP 86 (1970), 401-426.

Novakova, Iva, Sémantique du futur. Étude comparée français-bulgare, Paris, L'Harmattan, 2001.

Raible, Wolfgang, Von der Allgegenwart des Gegensinns (und einiger anderer Relationen). Strategien zur Einordnung semantischer Information, ZrP 97 (1981), 1-40.

Raible, Wolfgang, Comment intégrer la syntaxe dans la sémantique. La solution des grammairiens scolastiques, in: Lüdi, Georges/Stricker, Hans/Wüest, Jakob (edd.), «Romania ingeniosa». Festschrift für Prof. Dr. Gerold Hilty zum 60. Geburtstag. Mélanges offerts à Gerold Hilty à l'occasion de son $60^{\circ}$ anniversaire, Bern et al., Lang, 1987, 497-510.

Raynaud de Lage, Guy, Introduction à l'ancien français, Paris, Sedes, ${ }^{12} 1975$.

Schrott, Angela, Futurität im Französischen der Gegenwart. Semantik und Pragmatik der Tempora der Zukunft, Tübingen, Narr, 1997 (= 1997a).

Schrott, Angela, Futur du passé im Französischen der Gegenwart, RJb 48 (1997), $41-68$ (= 1997b).

Schwarze, Jochen, Grundlagen der Statistik I, Herne/Berlin, Verlag Neue Wirtschaftsbriefe, ${ }^{4} 1988$.

Sundell, Lars-Göran, Le temps futur en français moderne, Uppsala, Almqvist \& Wiksell, 1991.

Togeby, Knud, Grammaire française, vol. 2: Les formes personnelles du verbe, publié par Magnus Berg, Ghani Merad et Ebbe Spang-Hanssen, Copenhague, Akademisk Forlag, 1982.

Vendler, Zeno, Linguistics in Philosophy, Ithaka/New York, Cornell University Press, 1967.

Voß, Werner, et al., Taschenbuch der Statistik, München/Wien, Fachbuchverlag Leipzig/Hanser, 2000.

Werner, Edeltraud, Rez. zu Sokol 1999, VR 60 (2001), 346-352.

Wunderli, Peter, Die Bedeutungsgrundlagen der romanischen Futurbildung, ZrP 85 (1969), 385-415.

Wunderli, Peter, Virtualität, Aktualisierung und die Futurperiphrasen, ZrP 86 (1970), 386-400 (= 1970a).

Wunderli, Peter, Nochmals zur Aktualisierung und den Futurperiphrasen, ZrP 86 (1970), 427-448 (= 1970b).

Regensburg

ANGELA SCHROTT 